\title{
Organic Broadband Terahertz Sources and Sensors
}

\author{
Xuemei Zheng*, Colin V. McLaughlin, P. Cunningham, and L. Michael Hayden \\ Department of Physics, University of Maryland, Baltimore County, MD 21250, USA
}

\begin{abstract}
We review recent research using organic materials for generation and detection of broadband terahertz radiation $(0.3 \mathrm{THz}-30 \mathrm{THz})$. The main focus is on amorphous electrooptic (EO) polymers, with semiconducting polymers, molecular salt EO crystals, and molecular solutions briefly discussed. The advantages of amorphous EO polymers over other materials for broadband $\mathrm{THz}$ generation (via optical rectification) and detection (via EO sampling) include a lack of phonon absorption (good transparency) in the $\mathrm{THz}$ regime, high EO coefficient and good phase-matching properties, and, of course, easy fabrication (low cost). Our $\sim 12-\mathrm{THz}$, spectral gap-free $\mathrm{THz}$ system based on a polymer emitter-sensor pair is an excellent demonstration of the advantages using of EO polymers. We also present a model that can predict the performance of a polymer-based $\mathrm{THz}$ system. Both the dielectric properties of an EO polymer and laser pulse related parameters are included in the model, making the simulations close to real conditions. From our modeling work, the roles the dielectric properties play in the $\mathrm{THz}$ generation and detection are clearly seen, providing us with a good guide to select and design suitable EO polymers in the future.
\end{abstract}

Keywords: Electrooptic Polymer, Nonlinear Optics, Terahertz, Far Infrared, Spectroscopy.

\section{CONTENTS}

1. Introduction $\ldots \ldots \ldots \ldots \ldots \ldots \ldots \ldots \ldots \ldots \ldots \ldots \ldots \ldots$

2. Generation, Detection, and Application of

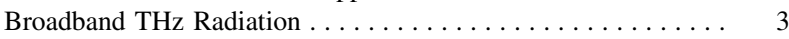

2.1. Basic Broadband $\mathrm{THz}$ System .............. 3

2.2. Broadband $\mathrm{THz}$ Generation ................. 3

2.3. Broadband $\mathrm{THz}$ Detection ............... 5

2.4. $\mathrm{THz}$ Time-Domain Spectroscopy ............. 6

3. Organic Materials for $\mathrm{THz}$ Sources and Detectors . . . . . . . . 6

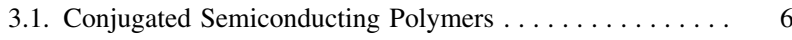

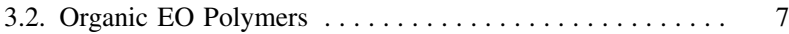

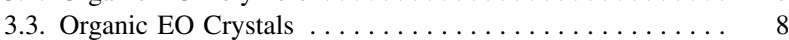

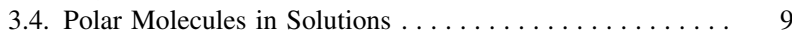

4. Experimental Details ...................... 9

4.1. Efficient $\mathrm{THz}$ Emitter Based on EO Polymer. . . . . . . . . . 9

4.2. LAPC Emitter-Sensor Pairs Operated at $\sim 800 \mathrm{~nm} \ldots . .99$

4.3. DAPC Emitter and Multi-Layer LAPC

Sensor Operated at $\sim 1300 \mathrm{~nm} \ldots \ldots \ldots \ldots \ldots \ldots \ldots$

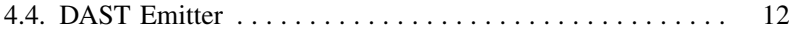

4.5. Dielectric Property Characterization of

EO Polymers Using THz-TDS . . . . . . . . . . . . 14

5. Modeling a Polymer Emitter-Sensor Pair . . . . . . . . . . . 15

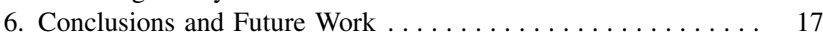

References and Notes .................... 18

\section{INTRODUCTION}

Terahertz $(\mathrm{THz})$ radiation, with a loose definition between $0.3 \mathrm{THz}$ and $30 \mathrm{THz}\left(1 \mathrm{THz}=10^{12} \mathrm{~Hz}\right)$, bridges microwave and infrared (IR) radiation. A variety of excitations,

\footnotetext{
*Author to whom correspondence should be addressed.
}

such as rotational and vibrational states in molecular systems, lattice resonance in dielectric crystalline materials, and confinement states in artificially fabricated nano-structures, occur in this spectral regime, suggesting the versatile application of the $\mathrm{THz}$ radiation in chemical and biological detection, medical imaging, and spectroscopy. However, the lack of compact, bright $\mathrm{THz}$ sources and sensitive $\mathrm{THz}$ detectors slows progress in this field. Even after intensive study for nearly two decades, THz science and technology is still in its infancy.

While tunable continuous-wave $\mathrm{THz}$ radiation, associated with photomixing or quantum cascade lasers, is useful for spectroscopy with very high frequency resolution, single-frequency imaging and remote sensing, pulsed (equivalently, broadband) $\mathrm{THz}$ radiation associated with the employment of ultrashort lasers is the optimal choice when an overall snapshot of the spectral characteristics of a sample in the THz regime is important. ${ }^{1}$ For pulsed $\mathrm{THz}$ systems, a wide bandwidth with a smooth frequency response using low power laser sources would be quite valuable in many scientific and technological arenas.

Currently, optoelectronic and all-optical techniques are commonly employed for generation and detection of pulsed $\mathrm{THz}$ radiation. The optoelectronic technique relies on the use of photoconductive dipole antennas (PDA) fabricated as micro-striplines or coplanar transmission lines on photoconductive inorganic substrates., ${ }^{2,3}$ These PDAs have excellent sensitivity and a smooth frequency response but a narrow useable bandwidth. The all-optical 
technique uses optical rectification $(\mathrm{OR})^{4}$ in electro-optic (EO) materials to generate the $\mathrm{THz}$ radiation and uses $\mathrm{EO}$ sampling ${ }^{5,6}$ to detect the $\mathrm{THz}$ radiation. This method has good sensitivity and a large bandwidth, but the conventional systems consisting of crystalline EO materials do not have a smooth frequency response across that bandwidth due in part to phonon absorption associated with the crystalline nature of the emitters and detectors.
Recently, some attention has been paid to the use of organic materials for the $\mathrm{THz}$ generation and detection. For both the optoelectronic and all-optical techniques, organic materials have shown great potential and broadened the material possibility. Organic EO materials have made significant contribution to the recent development of all-optical $\mathrm{THz}$ systems. For example, organic crystalline DAST (4- $N, N$-dimethylamino- $4^{\prime}-N^{\prime}$-methyl stilbazolium
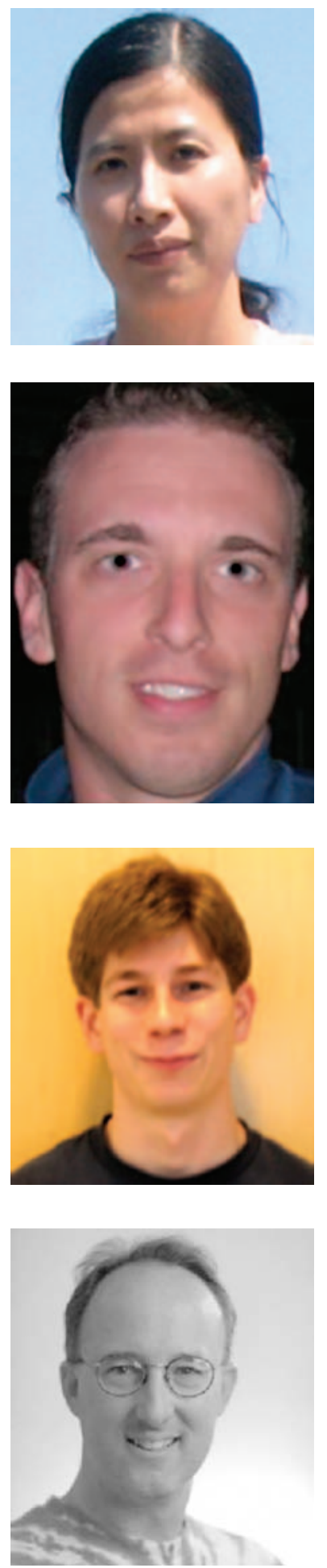

Xuemei Zheng is currently a postdoctoral research associate in the Physics Department at University of Maryland, Baltimore County. She received her Ph.D., MA, and BE from University of Rochester, the City College of New York, and Tianjin University (China), respectively. Her research interests involve terahertz optoelectronics, nonlinear optics in organic materials, and studies of ultrafast dynamics of photo-induced carriers in condensed matters.

Colin V. Mclaughlin was born in Portland, Maine, on July 30, 1979. He received his BA, majoring in physics, from Drew University in 2003. He received his MS from University of Maryland, Baltimore Co. in 2005. His research interests include EO polymer devices for $\mathrm{THz}$ applications.

P. Cunningham was born in Baltimore, MD April 29, 1982. He received his BS in applied physics from Towson University in 2004 and his MS from University of Maryland, Baltimore Co. in 2006. His research interests include optical-pump THz-probe studies of photoconductive materials and devices for solar cell and photodetector applications.

L. Michael Hayden is a Professor of Physics and the Chairman of the Department of Physics at the University of Maryland, Baltimore County. He has a BS from the U. S. Naval Academy and a Ph.D. from the University of California, Davis. He has had careers in the US Navy, the private sector, and academia. His current research interests involve ultra-fast optical studies of organic and polymeric materials, with applications to terahertz science. $\mathrm{He}$ is a private pilot with single and multi-engine ratings. 


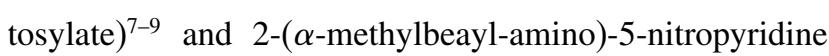
(MBANP). ${ }^{10}$ have been shown to exhibit much higher EO coefficients than their inorganic counterparts and efficiently generate and detect the $\mathrm{THz}$ radiation. More encouragingly, amorphous EO polymers exhibit not only high EO coefficients but also the absence of phonon absorption (in the $\mathrm{THz}$ regime). A $\mathrm{THz}$ system based on a polymer emitter-sensor pair has produced spectral gap-free bandwidth up to $\sim 12 \mathrm{THz} .{ }^{11}$ The most exciting thing about EO polymers is the tunability of the properties through composite constituent modification and film processing. This should allow these materials to establish excellent sensitivities, extremely wide bandwidths and flat frequency responses in the mid- and far-IR THz regimes. With slower progress, organic PDAs based on PPV ${ }^{12}$ and pentacene ${ }^{13}$ have also been successfully made for the $\mathrm{THz}$ emission.

Compared with a large amount of work done with inorganic crystalline materials, the use of organic materials in the $\mathrm{THz}$ generation and detection is still under-explored. With the great potential that the organic materials have shown, there is a need, at this point, to review $\mathrm{THz}$ sources and sensors involved with organic materials. In order to make this review more readable to researchers who are not quite familiar with but want to get involved in the $\mathrm{THz}$ science and technology, we will give an introduction in Section 2 to the common techniques used for $\mathrm{THz}$ generation and detection. We mainly focus on the all-optical technique involved with EO materials, taking our experience into consideration. Principles of $\mathrm{THz}$ time-domain spectroscopy (THz-TDS), one of the most important applications of $\mathrm{THz}$ radiation, are also presented in this section. Section 3 goes over organic materials for $\mathrm{THz}$ sources and sensors. Our focus is on amorphous EO polymers, but we also briefly discuss organic semiconductors and organic EO crystals and liquids as a complete review of this field. In Section 4, we present the experimental results obtained from our $\mathrm{THz}$ systems based on EO polymers operated at both $800-\mathrm{nm}$ and $\sim 1300 \mathrm{~nm}$ wavelength. Advantages of using EO polymers as $\mathrm{THz}$ emitters and sensors are clearly shown in this section. Experimental results using DAST as the THz emitter are also given in this section. Our modeling work on a polymer emitter-sensor pair is presented in Section 5. In Section 6 we conclude and point out challenges and future work.

\section{GENERATION, DETECTION, AND APPLICATION OF BROADBAND THz RADIATION}

\subsection{Basic Broadband THz System}

Broadband $\mathrm{THz}$ generation is intimately tied to femtosecond laser sources. In fact, $\mathrm{THz}$ technology boomed shortly after solid-state femtosecond lasers became widely available a little more than two decades ago that could be operated by a relative novice. Yet, compared to other spectral ranges of electromagnetic radiation, it is still quite a recent technology and not fully established.

A schematic of the optical arrangement of a THz system is shown in Figure 1. An output laser beam from a femtosecond laser is split into two beams, with most power going to the pump beam to drive a $\mathrm{THz}$ emitter and very little power going to the probe beam that interacts with the $\mathrm{THz}$ wave under investigation in a $\mathrm{THz}$ detector. By varying the optical delay line in one arm, the probe pulse (with duration much shorter than the $\mathrm{THz}$ pulse) sees different parts of the $\mathrm{THz}$ waveform. With the delay line position and data acquisition controlled by a computer, we can map out the electric field (instead of power) of the $\mathrm{THz}$ wave. This gated sampling technique allows for jitter-free phase coherent detection, leading to a high signal-to-noise ratio (SNR) and dynamic range.

\subsection{Broadband THz Generation}

In general, broadband $\mathrm{THz}$ radiation can be generated either in an optoeletronic manner involving photogenerated transient currents in photoconductive antennas. ${ }^{14}$ or in an optical manner involving optical rectification in EO materials. ${ }^{15}$ The two techniques have always been under parallel developments and boasted of different advantages.

The technique of using photoconductive antennas to generate electromagnetic radiation can be traced back to as early as the middle of the 1970's when Auston generated picosecond microwave pulses on a transmission line by exciting the photoconductor gap bridging the electrodes of the transmission line with picosecond pulses ${ }^{2}$ (see Fig. 2). The mechanism is simple: the optical pulse with the photon energy higher than the bandgap of the photoconductor excites electrons from the valance band to the conduction band; because of the electric field provided by the bias across the electrodes, the injection of these photocarriers closes the switch with the current through the switch rising rapidly (determined by the laser pulse duration) and decaying with a time constant determined by the carrier lifetime of the photoconductor; according to Maxwell's equations, $E(t) \propto \partial J(t) / \partial t$, so the transient photocurrent $J(t)$ radiates into the free space. ${ }^{16}$

The big step from microwave radiation to $\mathrm{THz}$ radiation was made possible by the availability of single-picosecond

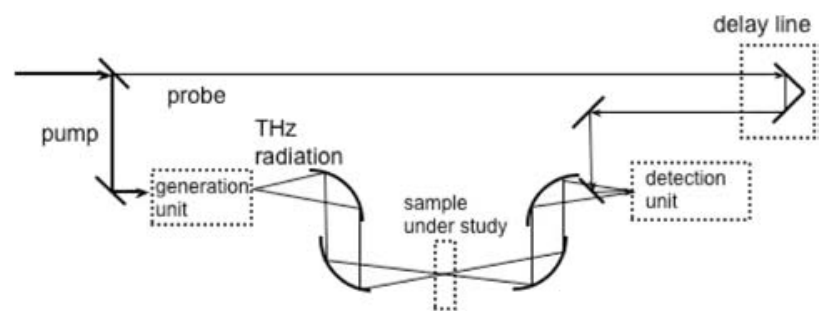

Fig. 1. General optical arrangement of a $\mathrm{THz}$ system. Four $90^{\circ}$ offaxis parabolic mirrors are used to collect, collimate and focus the $\mathrm{THz}$ radiation. This arrangement is suitable for spectroscopy study, for which a sample under study can be placed at the $\mathrm{THz}$ focal point. 


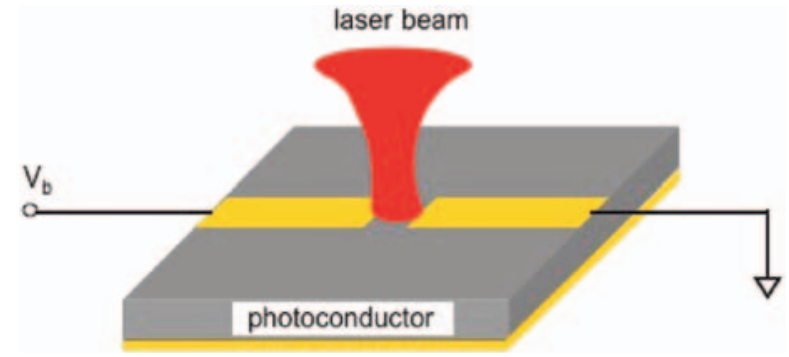

Fig. 2. A photoconductive switch integrated in a microstrip transmission line. When a laser pulse with photon energy higher than the photoconductor's bandgap energy illuminates the gap of the transmission line biased by an external field, photogenerated carriers close the switch and the transient current consequently radiates into free space.

and subpicoscecond photoconductors (LT-GaAs, ionimplanted GaAs, etc.), micro-lithography (allowing fabrication of smaller radiating devices and consequently higher frequency electromagnetic radiation), and ultrashort lasers providing shorter and shorter pulses (down to $\sim 12$ fs, commercially available). The achievable bandwidth from most PDA-based $\mathrm{THz}$ systems is usually a few $\mathrm{THz}$ and is often attributed to the limit of the carrier lifetime of the photoconductor involved. In a few cases, however, ultrabroad bandwidths $(>10 \mathrm{THz})$ have been reported. Kono et al. ${ }^{17}$ reported a THz detection up to $20 \mathrm{THz}$ with a low-temperature-grown GaAs (LT-GaAs) PDA gated with 15 fs light pulse. It was quite a surprising result as the LT-GaAs they used exhibited a relatively long carrier lifetime of $\sim 1.4 \mathrm{ps}$. According to the authors, the fast response of the PDA was explained by the fast rise in the photocurrent upon excitation by the ultrashort laser pulse, ${ }^{17}$ and the physical origin of the fast photocurrent within $\sim 100$ fs might be explained by the ballistic transport of the photoexcited electrons in the biased electric field. ${ }^{18}$ In this picture, the PDA works as an integration detector, so the photocurrent from the antenna should be proportional to the time integration of the incident $\mathrm{THz}$ radiation. With a post-measurement analysis where both the number of photocarriers (a function of time) and the integration mode of the PDA detector were taken into consideration, the same authors obtained even broader detection bandwidth, up to $\sim 40 \mathrm{THz}^{19}$ On the other hand, ultrabroadband $\mathrm{THz}$ generation from LT-GaAs PDAs was demonstrated by Shen et al. ${ }^{20}$ Using a backward collection scheme to minimize the $\mathrm{THz}$ absorption by the LTGaAs substrate, $\mathrm{THz}$ radiation with frequency components over $30 \mathrm{THz}$ was achieved, and the transverse optical (TO) phonon absorption band of GaAs was clearly identified. The ultrabroad bandwidth might be due to the specific scheme where the pump beam was illuminated on the edge of one of the PDA electrodes. ${ }^{21-23}$ For the edge illumination scheme, the transient current in the PDA results from the dielectric relaxation of the space-charge field such that its dynamics is not determined by the carrier lifetime. ${ }^{24}$ So far, the broadest bandwidth from a PDA emitter-sensor pair is $\sim 15 \mathrm{THz},{ }^{25}$ although a very distinguished spectral gap related to the TO phonon band of the substrate was exhibited. In addition to the operation complexity, another disadvantage of this technique is the necessity of the complex and expensive lithography facility.

Compared with the technique involved with PDAs, the advantage of using nonlinear optical rectification to generate $\mathrm{THz}$ radiation is a possible broader bandwidth, as well as the availability of a variety of EO materials. Pioneering work done by Shen et al. ${ }^{4}$ demonstrated the possibility of using picosecond laser pulses in EO materials to generate far infrared radiation via optical rectification. Auston et al. extended this technique by using shorter laser pulses and observed a generated electromagnetic wave in the $\mathrm{THz}$ regime. ${ }^{26}$ Since then, many researchers have followed and further developed this technique by exploiting numerous materials and geometries. ${ }^{7,15,27-29}$

Optical rectification can be understood as mixing of two different frequency components in the frequency spectrum of an incident ultrashort optical pulse in an EO medium. The difference frequency mixing results in a nonlinear polarization and consequently a radiation at the beat frequency. The bandwidth of the radiation in OR is limited by the bandwidth of the optical pulse, as well as the relevant properties of the nonlinear medium. Mathematically, the difference frequency mixing process via optical rectification is describe as follows:

$$
\left[\frac{d^{2}}{d z^{2}}+\varepsilon(\Omega) \frac{\Omega^{2}}{c^{2}}\right] \widetilde{E}_{\mathrm{THz}}(z, \Omega)=\frac{4 \pi}{c^{2}} \Omega^{2} P_{\mathrm{NL}}(z, \Omega)
$$

where $c$ is the speed of light, $\Omega$ is the THz frequency, $\varepsilon(\Omega)$ is the dielectric constant of the nonlinear medium in the THz region $\left[\varepsilon(\Omega)=n_{\mathrm{THz}}^{2}(\Omega)\right.$, if there does not exist $\mathrm{THz}$ absorption in the NLO medium], $\widetilde{E}_{\mathrm{THz}}(z, \Omega)$ is the propagating $\mathrm{THz}$ field generated in the nonlinear medium, and $P_{\mathrm{NL}}(z, \Omega)$ is the nonlinear polarization propagating along the $z$-axis expressed by (assuming the optical wavelength is far away from the material's electronic resonance region):

$$
\begin{aligned}
P_{\mathrm{NL}}(z, \Omega) & =\chi_{\mathrm{eff}}(\Omega) \int_{-\infty}^{\infty} E(z, \omega) \cdot E(z, \omega-\Omega) d \omega \\
& =\chi_{\mathrm{eff}}(\Omega) \cdot I(\Omega, z)
\end{aligned}
$$

where $\omega$ is the optical frequency. It should be noted that $I(z, \Omega)$ is the autocorrelation of the optical electric field, or, actually, the Fourier transform of the intensity profile of the optical pulse- $I(z, t)$. Dispersion and absorption of the nonlinear medium in both the $\mathrm{THz}$ and optical regime make analytically solving Eq. (1) very difficult, if not impossible. However, analytical solutions based on certain assumptions simplifying the problem can be obtained. ${ }^{30}$ It is found, from these solutions, that the phase-mismatch (the difference between the optical group index $n_{g}=n_{\text {opt }}-$ $\lambda_{\text {opt }} \frac{d n_{\text {opt }}}{\lambda_{\text {opt }}}$, where $n_{\text {opt }}$ and $\lambda_{\text {opt }}$ are the optical index and optical wavelength, respectively, and the THz index $n_{\mathrm{THz}}$ in the material) limits the amplitude and bandwidth of the 
$\mathrm{THz}$ generation. In fact, the desired phase-matching condition $n_{g}=n_{\mathrm{THz}}$ can also be identified by carefully scrutinizing Eq. (1): the nonlinear polarization involves the optical group index while the $\mathrm{THz}$ electric field involves the $\mathrm{THz}$ index. Alternatively, the phase-matching requirement can be derived by resorting to a quantum picture. ${ }^{31}$ As is well known, all particles involved in an interaction must obey the laws of both energy conservation and momentum conservation. There are two optical photons (with the energy difference being the $\mathrm{THz}$ photon energy) and one $\mathrm{THz}$ photon in the OR process, so the phase-matching condition is: $\Delta k=k_{\text {opt }}(\omega-\Omega)-k_{\text {opt }}(\omega)+k_{\mathrm{THz}}(\Omega)=\Omega\left[n_{g}(\omega)-\right.$ $\left.n_{T H z}(\Omega)\right] / c=0$. In the case of phase-mismatching $(\Delta k \neq$ $0)$, the coherence length (optimal interaction length) can be expressed as: ${ }^{31}$

$$
l_{c}=\frac{\pi}{\Delta k}=\frac{\pi c}{\Omega\left(n_{g}-n_{\mathrm{THz}}\right)}
$$

Clearly, better phase-matching leads to a longer coherence length and potentially greater $\mathrm{THz}$ generation.

Another issue related to the optical manner of generating $\mathrm{THz}$ radiation is the phonon absorption that is associated with the lattice resonance effect in an EO crystal. Absorption of the $\mathrm{THz}$ radiation (or, spectral gaps) is the direct result of this effect. For example, $\mathrm{ZnTe}$, a standard $\mathrm{EO}$ crystal for $\mathrm{THz}$ generation, exhibits a TO phonon resonance at $\sim 5.3 \mathrm{THz} .{ }^{32}$ In addition, a large dispersion of the $\mathrm{THz}$ index exists near any absorption band. This can have an significant influence on the phase matching. We still take $\mathrm{ZnTe}$ as the example. Its wide employment in the $\mathrm{THz}$ generation (and detection) is mostly due to the fact that there exists good phase-matching at $\sim 2 \mathrm{THz}$ for the optical pump wavelength of $\sim 800 \mathrm{~nm}$ (the emission wavelength of widely available femtosecond Ti: sapphire lasers). However, the dispersive nature of $\mathrm{THz}$ index in $\mathrm{ZnTe},{ }^{33}$ makes the good phase-matching region very narrow. For this reason, in order to achieve broadband $\mathrm{THz}$ emission, a very thin ZnTe crystal is essential, making the conversion efficiency low and the cost of the crystal preparation high. As will be discussed in the Section 3, organic EO polymers do not have lattice structures and consequently do not have phonon absorption and serious $\mathrm{THz}$ index dispersion problems, suggesting their promising role as broadband $\mathrm{THz}$ emitters.

Compared with PDAs, the optical to $\mathrm{THz}$ conversion efficiency is low in EO materials. However, when a laser amplifier is used with EO materials, achievable SNR is comparable to the case where a PDA is used with a laser oscillator. For the later, the high excitation density associated with an amplified pulse is not beneficial since electron screening effects give an adverse effect on the $\mathrm{THz}$ emission leading to lower amplitude and bandwidth; while for the nonlinear OR effect, the $\mathrm{THz}$ amplitude is proportional to the optical pump intensity such that below the material's damage threshold, the higher the pump intensity, the more $\mathrm{THz}$ emission.

\subsection{Broadband THz Detection}

Like $\mathrm{THz}$ generation, $\mathrm{THz}$ detection can be done in an optoelectronic manner called photoconductive sampling or an optical manner called EO sampling.

Historically, the appearance of photoconductive sampling was almost as early as the first use of a PDA to generate electromagnetic pulses. ${ }^{34} \mathrm{~A}$ pair of PDAs and a femtosecond laser make the heart of an optoelectronic $\mathrm{THz}$ system. To operate a PDA detector, no DC bias is applied across the device. Instead, the $\mathrm{THz}$ pulse under measurement provides the electric field to accelerate the photogenerated carriers resulting from a optical probe pulse that overlaps with the $\mathrm{THz}$ pulse spatially and temporally. It should be kept in mind that it is the $\mathrm{THz}$ waveform that one wants to measure. Indeed, the varying electric field of the $\mathrm{THz}$ waveform determines the amplitude of the photocurrent that is measured by an ampere meter. By varying the delay time, the $\mathrm{THz}$ waveform is mapped out.

EO sampling, as an alternative to photoconductive sampling, was first established by Valdmanis et al. ${ }^{35}$ as a tool to characterize ultrashort electrical pulses in high-speed circuits, which could not possibly be done by any oscilloscope. EO sampling then had a picosecond temporal resolution, limited by available picosecond lasers. With the era of femtosecond lasers coming in the middle of the 1990's, the temporal resolution of EO sampling was also shortened to the femtosecond scale, much faster than any available oscilloscope. A variety of EO crystals and experimental configurations. ${ }^{36,37}$ have been employed in order to make EO sampling more compatible with the device under test and to improve the measurement sensitivity, dynamic range, and response time such that smaller and shorter electrical signals obtained from "exotic" devices can be measured. For a different purpose, free space EO sampling ${ }^{5}$ was developed and is now widely employed to detect freely propagating $\mathrm{THz}$ radiation.

The building block of EO sampling is indeed an EO intensity modulator (see Fig. 3). When a linearly polarized optical beam goes through an EO crystal, the electric-field induced birefringence in the crystal changes its polarization state. This polarization change is then detected by an polarization analyzer that converts this change into an intensity change detectable by a photodiode. When EO sampling is used to detect electrical pulses in a device, the electric field comes from the guided electrical pulses. When it is used to detect free-space $\mathrm{THz}$ radiation, the electric field is from a $\mathrm{THz}$ pulse. The waveplate is used to optically bias the static phase retardation at $\sim \pi / 2$ such that the measurement is done in the most linear regime leading to the least signal distortion. ${ }^{36}$

Because this paper is about $\mathrm{THz}$ technology, we will only focus on free-space EO sampling. Assuming that the EO response is instantaneous and the amplitude of the $\mathrm{THz}$ waveform is not very large such that the EO detection works within its linear regime and considering the usual 


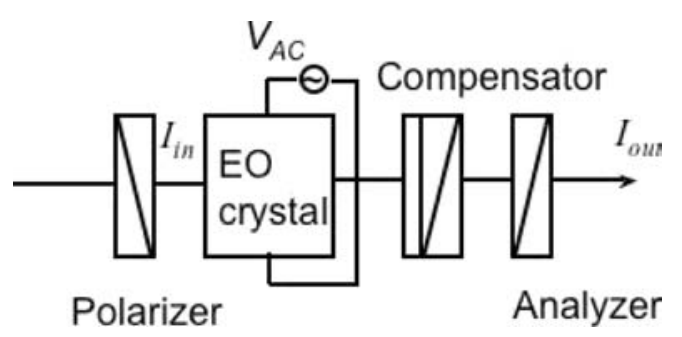

Fig. 3. Schematic of an EO intensity modulator. The analyzer is set cross to the polarizer. For this schematic, the output power $I_{\text {out }}$ is related to the incident power $I_{\text {in }}$ through $I_{\text {out }}=I_{\text {in }} \sin ^{2}\left[\left(\Gamma_{o}+\pi V_{\mathrm{AC}} / V_{\pi}\right) / 2\right]$, where $\Gamma_{o}$ is the phase retardation due to the intrinsic birefringence of both the EO crystal and the compensator, and $V_{\pi}$ is the characteristic half-wave voltage corresponding to the EO crystal. In order to operate the modulator in the most linear regime, $\Gamma_{o}$ should be set at $\pi / 2$ (by adjusting the compensator). In this case, $I_{\text {out }}=I_{\text {in }}\left[1 / 2+1 / 2 \sin \left(\pi V_{\mathrm{AC}} / V_{\pi}\right)\right]$. If $V_{\mathrm{AC}}$ is very small, then the AC output $\left(I_{\text {out }}\right)_{\mathrm{AC}} \propto I_{\text {in }} \sin \left(\pi V_{\mathrm{AC}} / V_{\pi}\right) \propto I_{\text {in }} V_{\mathrm{AC}}$, i.e., the signal is approximately linear with the external voltage.

configuration of collinear propagation of $\mathrm{THz}$ and optical probe pulses, the signal measured is given by

$$
\begin{aligned}
S(\tau) & \propto \int_{0}^{d} \int_{-\infty}^{\infty} \Delta n(z, t) I_{\mathrm{opt}}\left(t-n_{g} z / c-\tau\right) d t d z \\
& \propto \int_{0}^{d} \int_{-\infty}^{\infty} E_{\mathrm{THz}}(z, t) I_{\mathrm{opt}}\left(t-n_{g} z / c-\tau\right) d t d z
\end{aligned}
$$

where $\Delta n(z, t)$ is the birefringence induced by the local transient electric field, $\tau$ is the delay time between the $\mathrm{THz}$ and optical pulse, $d$ is the interaction length, and $I_{\text {opt }}(t)$ is the intensity profile of the optical probe pulse that propagates at its group velocity and temporally delayed with regard to the $\mathrm{THz}$ pulse.

Ideally, $S(\tau)$ should honestly follow the change of $E_{\mathrm{THz}}$ for an undistorted measurement. In reality, even assuming that the EO material itself does not change the incident $\mathrm{THz}$ waveform and the probe pulse profile and that the measurement is in the linear regime, there still are two factors that distort the measured $\mathrm{THz}$ waveform. The first factor is the finite laser pulse duration. From Eq. (3) it can be seen that $S(\tau)$ is actually the convolution of the $\mathrm{THz}$ pulse and the probe pulse. If the probe pulse is much shorter than the $\mathrm{THz}$ pulse then we do not have to resort to the devolution to extract the true $\mathrm{THz}$ waveform. This is generally true in most cases. The other factor is phase-mismatch, which can be more easily identified in the frequency domain. For this purpose, we perform a Fourier transform on Eq. (3). With THz field spectrum expressed as $E_{\mathrm{THz}}(z, \Omega)=E(\Omega) \exp \left(i \Omega n_{\mathrm{THz}} z / c\right)$ (ignoring $\mathrm{THz}$ absorption and index $n_{\mathrm{THz}}$ dispersion in the EO material) and the Fourier transform of the propagating pulse as $I(\Omega) \exp \left(-i \Omega n_{g} z / c\right)$, it can easily be found that

$$
S(\Omega) \propto E(\Omega) I(\Omega) \int_{0}^{d} \exp \left[i \Omega\left(n_{\mathrm{THz}}-n_{g}\right) z / c\right] d z
$$

Therefore, the phase-matching requirement is $n_{g}=n_{\mathrm{THz}}$, exactly the same as in the case of the $\mathrm{THz}$ generation via OR. If the phase-matching is not satisfied in an EO sampling measurement, the collinearly propagating $\mathrm{THz}$ and optical pulse will gradually walk off from each other. The walk-off effect distorts the measured signal. With the presence of phase-mismatching, the larger the thickness of the EO material, the worse the signal distortion will be. It should be pointed out that a real situation can be even more complicated since most EO materials have not only dispersive $\mathrm{THz}$ index but also frequency-dependent $\mathrm{THz}$ absorption, leading to a distortion of the propagating $\mathrm{THz}$ wave with the distance. In the material section below, we will discuss the advantage of using EO polymers which have no phonon absorption and the related $\mathrm{THz}$ index dispersion.

\subsection{THz Time-Domain Spectroscopy}

Among many $\mathrm{THz}$ applications, $\mathrm{THz}$ time-domain spectroscopy (THz-TDS) is the most widely explored. THz-TDS is an important method for spectroscopic investigations of dielectric materials and is considered superior to other methods (Fourier Transform IR spectroscopy or the use of free electron lasers) due to the high brightness of the source, coherent, low background detection, and the ability to determine real and imaginary parts of the dielectric function without resorting to Kramers-Kronig relations. Recently, THz-TDS has been expanded to help characterize artificially structured materials such as nanoparticles $^{38}$ and metamaterials ${ }^{39}$ that have attracted strong attention among researchers with backgrounds varied from electromagnetics, optics, engineering, chemistry, and physics, to materials science. Reviews of the field of THzTDS, its sources, sensors, and techniques are given by Grischkowsky, ${ }^{40}$ Nuss, ${ }^{16}$ and more recently, Mittleman. ${ }^{41}$

For all linear spectroscopy systems, the possibility of studying a material relies on the interaction between the material and the electromagnetic radiation. For THz-TDS, this radiation is in the THz regime. A typical THz-TDS system is just like Figure 1. Generally, two measurements are required: the first one measures $E_{\text {ref }}(t)$ without the sample or with a sample of known dielectric properties, and the second one measures $E_{\text {sample }}(t)$ in which the THz radiation interacts with the sample inserted at the focal point of the $\mathrm{THz}$ path (see Fig. 1). The two time-domain waveforms are then Fourier transformed to frequency domain. Comparing $E_{\text {ref }}(\Omega)$ and $E_{\text {sample }}(\Omega)$, one can extract dielectric properties such as refractive index and absorption coefficient in insulators and the complex conductivity in metals, semiconductors and superconductors.

\section{ORGANIC MATERIALS FOR THz SOURCES AND DETECTORS}

\subsection{Conjugated Semiconducting Polymers}

As we have seen from the above discussion, the optoelectronic technique associated with PDAs requires photoconducting semiconductors. Photoconductivity exists not only 
in inorganic semiconductors but also in conjugated semiconducting polymers. Since Shirakawa et al. first reported conductivity in polyaceylene, ${ }^{42}$ a large amount of research has been carried out to understand the material properties of the family of conjugated polymers. The drive behind this extensive effort is the possibility of electronic and photonic devices that are of low cost, easy fabrication, and tunable properties. Specifically, for $\mathrm{THz}$ applications, one is likely to ask whether photoconductive polymers are suitable materials for PDAs and can eventually replace inorganic photoconductors. Indeed, $\mathrm{THz}$ radiation has been obtained from a poly(p-phenylene cinylene) $(\mathrm{PPV})^{12}$ PDA and a pentecene ${ }^{13}$ PDA. However, further advancement will depend on significant improvement of the relevant properties (such as mobility and lifetime) in the semiconducting polymers. At this point, there are several issues of concern. Some fundamental physics underlying the construction and optimization of these devices still remain controversial or poorly understood. For example, there exists disagreement on carrier photoexcitation in conjugated polymers, with some evidences supporting the interband photoexcitation picture. ${ }^{43-47}$ and others supporting the exciton dissociation picture. ${ }^{4-50}$ There also exists a dramatic difference in the magnitude of the measured mobility. Since mobility is an important concept in understanding and improving the device performance, an accurate measurement is essential. Ongoing research on these issues should help remove the obstacles of using conjugated semiconducting polymers in this field.

\subsection{Organic EO Polymers}

The first work using poled EO polymers to generate and detect the $\mathrm{THz}$ radiation was done by Nahata et al. ${ }^{6,27}$ As mentioned above, the intrinsic lattice resonance and generally existing phase-mismatch in inorganic EO materials prevents generation and detection of the spectral gapfree, broadband THz radiation. Amorphous EO polymers, instead, do not have a lattice structure and have a quite flat refractive index ranging from NIR to FIR. The latter property suggests the possibility of achieving good phase matching, which would lead to broadband $\mathrm{THz}$ generation and detection. In addition, EO polymers have advantages over inorganic materials with regard to fabrication ease and low cost. Above all, maybe the most exciting thing about using EO polymers is the material tunability that can lead to suitable properties for specific applications.

To design and build an EO polymer involves selection of an active component-NLO chromophore, a passive component-polymer matrix, and the physical connectivity between the two ${ }^{51}$ Therefore, to tune the properties of an EO polymer, one also has to consider the three factors (mostly, the first two). To make our EO polymers, we have been using a guest (EO chromophore)-host (polymer) configuration, as it offers the greatest ease in the design and availability of the components. We focused our attention on selecting suitable NLO chromophores and polymers. The guest NLO chromophore can be chosen from a wide range of known systems with a range of established physical properties such as absorption spectra $\left(\lambda_{\max }\right.$ and cutoff), dipole moments (ground state $\mu_{\mathrm{g}}$ and excited state $\mu_{\mathrm{e}}$ ), and the second-order molecular optical polarizability (the frequency dependent $\beta$ ). A more attractive approach, however, is to modify chromophores to fit the applications. As for polymers, a well-established and commercially available group now exists with suitable physical properties. Sufficiently high glass transition temperature $\left(T_{\mathrm{g}}\right)$, capability of processing into films, and matching linear optical and $\mathrm{THz}$ properties (absorption and refractive index) should all be considered when choosing a suitable polymer.

In reality, the physical compatibility between the chromophore and polymer must also be considered such that a sufficient amount of the chromorphore can be added to the polymer to achieve the desire bulk EO coefficient without causing phase separation. It should be noted that the inclusion of the chromophore molecules into the polymer matrix reduces $T_{\mathrm{g}}$. For this reason, the intrinsic $T_{\mathrm{g}}$ of the chosen polymer should be high enough such that the EO polymer system ends up in a useful range for poling and stability. Electric field poling is an essential step during the fabrication of EO polymers. It is usually performed at or just below the $T_{\mathrm{g}}$ of the material, as the NLO chromophore molecules have to be mobile in order to be easily oriented by the poling field. The oriented NLO chromophore molecules break the centrosymmetric structure of the material so as to allow for bulk EO coefficients. A very high $T_{\mathrm{g}}$ can cause difficulty for a practical poling procedure, while a too low $T_{\mathrm{g}}$ can cause a rapid loss of poling order at the operation temperature (in our case, room temperature). Thus, a $T_{\mathrm{g}}$ falling into a suitable poling temperature range is very important.

Although the nonlinearity of the EO polymer is due to the active NLO chromophore and the electronic and linear optical properties are also mostly determined by it, these properties are affected to some degree by the passive polymer in the composite too. For example, the optical absorption and hyperpolarizability of the mixed EO composite are generally different from the corresponding NLO chromophore.

Among the many options of polymer hosts and NLO chromophores, we found that EO polymers consisting of an amorphous polycarbonate (APC) copolymer as the host and Lemke dye or DCDHF type dye as the guest worked the best with widely available Ti: sapphire lasers. APC is commercially available and forms high optical-quality films, with an intrinsic $T_{\mathrm{g}}$ at $205^{\circ}$ (sufficiently high to permit the addition of an adequate guest chromophores). Like all the members in the NLO chromophore family, Lemke and DCDHF type dye (see Fig. 4) are characterized by an electron-rich donor group, a conjugated linkage, and an electron-deficient acceptor group in each molecule. 

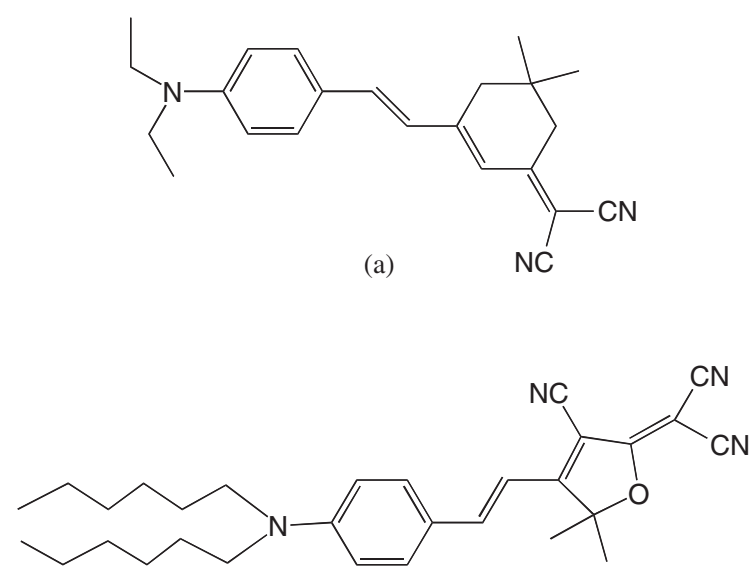

(b)

Fig. 4. Chemical structure of (a) Lemke and (b) DCDHF-6-V.

To make easy references, we name the EO polymer based on Lemke as LAPC and that based on DCDHF as DAPC.

To make LAPC (40\% Lemke/60\% APC) and DAPC (40\% DCDHF/60\% APC) films, ${ }^{51-53}$ the first step is dissolving each component in dichloroethane in a $10 \%$ solids/solvent ratio. The films are then cast from the solution onto glass substrates coated with indium tin oxide (ITO). After solvent evaporation, two solid polymer films are pressed in vacuum $70{ }^{\circ} \mathrm{C}$ above its glass transition temperature $T_{\mathrm{g}}$ for $10 \sim 15$ minutes. The thickness of the resulting film is controlled by appropriate polyimide spacers. High-optical-quality films with thickness in the range of 50-350 $\mu \mathrm{m}$ can be obtained with this method. After the film making, sufficient electric poling is performed so as to achieve high EO coefficients. A poling field as high as $\sim 100 \mathrm{~V} / \mu \mathrm{m}$ can routinely be applied without causing breakdown in the polymers. At this level of poling, $r_{33}>$ $50 \mathrm{pm} / \mathrm{V}$ and $r_{33}>30 \mathrm{pm} / \mathrm{V}$ at $785 \mathrm{~nm}$ can be achieved for DAPC and LAPC, respectively. Because a THz wave does not penetrate the conductive ITO coatings very well, it is important to remove at least one substrate such that the ITO coating will not interact with the $\mathrm{THz}$ wave. In the case that laser pulses are very short ( $<50$ fs FWHM), the other substrate should also be removed to obtain freestanding films such that the laser pulse duration is not broadened.

\subsection{Organic EO Crystals}

The most widely studied organic EO crystal for $\mathrm{THz}$ applications is DAST, which has optical absorption peaks at $\sim 530 \mathrm{~nm}$ for $a$-polarized radiation and at $\sim 500 \mathrm{~nm}$ for $b$ polarized radiation. Using DAST to generate $\mathrm{THz}$ radiation dates back to $1992 .^{7}$ The very efficient $\mathrm{THz}$ emission in this experiment was attributed to the high nonlinear coefficients of DAST. However, $\mathrm{THz}$ detection using DAST ${ }^{54}$ was not reported until 2000, probably because high quality crystals were not accessible. In that work, modified
EO detection using a double-pass in the DAST crystal was employed to overcome the high birefringence of the crystal. Lately, Schneider et al. ${ }^{55}$ introduced a technique utilizing a THz-induced lensing effect in DAST for $\mathrm{THz}$ detection. Compared with the former modified EO detection, the THz-induced lensing effect involved less optical elements in the set-up and led to an easier alignment procedure, eliminating obstacles associated with use of EO materials exhibiting high birefringence. Eventual commercialization of high optical-quality DAST crystals was mostly credited to the research conducted by Gunter's group at Institute of Quantum Electronics, ETH Zurich, in Switzerland. ${ }^{56-58}$

To use DAST in THz generation and detection, knowledge of its linear and nonlinear optical properties and linear $\mathrm{THz}$ properties is important. The past ten years have seen a large amount of work done in characterization of the optical properties of DAST, where polarization dependent optical absorption, refractive index, and nonlinear coefficients were measured. ${ }^{56} \mathrm{THz}-\mathrm{TDS}$ and infrared spectroscopy were employed to study the dielectric properties, such as absorption and refractive index, of DAST in the $\mathrm{THz}$ regime. The THz-TDS study done by Walther et al. ${ }^{59}$ only showed spectroscopic results up to $\sim 3 \mathrm{THz}$, restricted by the authors' available $\mathrm{THz}$ sources and detectors. While capable of providing a wealth of information in the mid-IR regime, the infrared spectroscopic study done by Bosshard et al. ${ }^{60}$ does not have enough sensitivity to identify absorption bands due to weak lattice vibrations (resulting from small intermolecular interactions) below $12 \mathrm{THz}$.

Very recently, based on the knowledge from previous material property studies, Schneider et al. conducted detailed investigations on the application of DAST in the $\mathrm{THz}$ generation and detection, ${ }^{55,61-63}$ including issues such as $\mathrm{THz}$ and optical absorption and polarization dependent phase-matching. These studies focused on two pump wavelength ranges, one associated with a Ti:sapphire amplifier system emitting at $\sim 800 \mathrm{~nm}$ and the other with an optical parametric amplifier with a tuning range from $\sim 1200 \mathrm{~nm}$ to $\sim 1500 \mathrm{~nm}$. These papers should be used as a guide for anyone having intentions to employ DAST for THz applications.

It should be pointed out that DAST has also been used as a nonlinear optical crystal for differential frequency generation involving two pump beams with different wavelengths. ${ }^{64-66}$ By tuning the wavelength of one pump beam, THz radiation can be tuned too. In this way, a wide range of $\mathrm{THz}$ frequencies were achieved from a DAST crystal and multiple $\mathrm{THz}$ absorption bands were identified.

Being less investigated, the organic molecular crystal MBANP was also used for the $\mathrm{THz}$ generation. ${ }^{10}$ This material is highly absorptive for all wavelengths below $450 \mathrm{~nm}$ and exhibits an EO coefficient $r_{\text {eff }}=18.2 \mathrm{pm} / \mathrm{V}$ at $632.8 \mathrm{~nm}$. In the reported experiment, a $\sim 200-\mu \mathrm{m}$-thick $\langle 001\rangle$ MBANP crystal was pumped by both $800-\mathrm{nm}$ and 
$400-\mathrm{nm}$ femtosecond pulses. The generation of $\mathrm{THz}$ radiation in the first case is via OR, and in the other case is via a direct intramolecular charge transfer.

\subsection{Polar Molecules in Solutions}

Fundamentally, $\mathrm{THz}$ generation is always associated with a charge transfer of some kind. For PDAs, it is the photogenerated carriers dynamics in the biased field. For inorganic EO media, it is the deformation of the electron clouds of atoms and ion vibration; while for organic EO media, it is the intramolecular electron transfer between donor and acceptor sites. Based on this reasoning, Beard et al. ${ }^{67}$ designed and conducted a very interesting experiment, generating $\mathrm{THz}$ radiation from a Betaine-30 solution (with $\mathrm{CHCl}_{3}$ solvent). In order to orient the Betaine-30 molecules they applied a bias across the solution. Pulses from a ultrashort laser then photo-induced the charge transfer in the molecules leading to generation of $\mathrm{THz}$ radiation. The polarity of the $\mathrm{THz}$ waveform is determined by the charge transfer direction.

It should be pointed out that there is a similarity between the poling procedure during the making of EO polymers and the orientation of Betaine- 30 molecules in solution with a biasing voltage. For EO polymers, poling order is frozen even after the removal of the poling field, while for solutions, a real-time bias is needed. With a low- $T_{\mathrm{g}}$ EO polymer, real-time poling is also realistic. An advantage of real-time poling is that one can shape the $\mathrm{THz}$ waveform by varying the applied poling field (strength and polarity) at any time.

\section{EXPERIMENTAL DETAILS}

\subsection{Efficient THz Emitter Based on EO Polymer}

It is expected that $\mathrm{EO}$ polymers are more efficient $\mathrm{THz}$ emitters than $\mathrm{ZnTe}$, as the former has EO coefficients at least one order of magnitude higher than the latter. Considering the transverse poling geometry (poling field normal to the surface) and the $p$-polarization of the pump beam in our experiments, we need to orient the polymer film such that the pump beam is incident at Brewster's angle in order to achieve the maximal $\mathrm{THz}$ emission. Because of this geometry, roughly half of the magnitude of the nonlinearity of the EO polymers can be accessed. Even so, in our experiments with a 50-fs-duration, $800-\mathrm{nm}$-wavelength, $1-\mathrm{kHz}$, Ti:sapphire laser amplifying system (SpectraPhysics Spitfire) we found that an $80 \mu \mathrm{m}$ thick EO polymer film generates more $\mathrm{THz}$ emission (per optical pump photon) than a $1000-\mu \mathrm{m}$-thick ZnTe crystal (see Fig. 5)..$^{53}$

\subsection{LAPC Emitter-Sensor Pairs Operated at $\sim 800 \mathrm{~nm}$}

As pointed out in Section 2, the phase-matching condition determines the optimal interaction length (the coherence

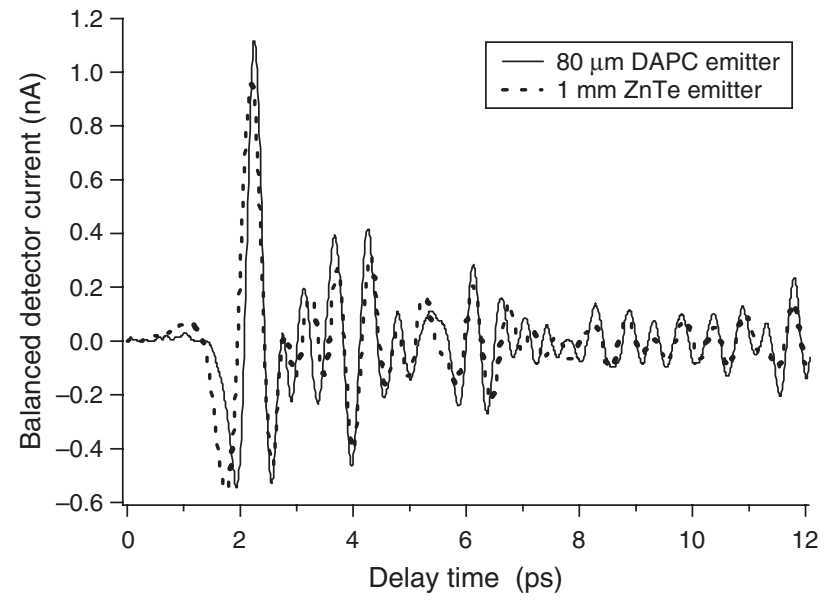

Fig. 5. Comparison of the THz field emitted from a $1 \mathrm{~mm}$ thick $\mathrm{ZnTe}$ crystal and an $80 \mu \mathrm{m}$ thick polymer layer (40\%DCDHF-6-V/60\%APC). The EO coefficient of the polymer is $47 \pm 2 \mathrm{pm} / \mathrm{V}$, in comparison to $\sim 4 \mathrm{pm} / \mathrm{V}$ for $\mathrm{ZnTe}$. The detection was done using EO sampling with a $2 \mathrm{~mm}$ thick ZnTe crystal.

length) for both $\mathrm{THz}$ emitters and sensors based on $\mathrm{EO}$ materials. Because the coherence length is dependent on frequency, phase-mismatch induced spectral dips are expected for either a thick emitter or a thick sensor or their combination. Our experiments clearly demonstrate this. Four layers of freestanding LAPC films with thicknesses controlled by $\sim 75-\mu \mathrm{m}$-thick spacers, and one layer of LAPC with a thickness controlled by a $\sim 120-\mu \mathrm{m}-$ thick spacer, were prepared using the method described in Section 3.2. The thick film was used as the $\mathrm{THz}$ sensor, while the other four were used as the $\mathrm{THz}$ emitter. By stacking the films up one by one, each oriented with the same poling polarity, we obtained multi-layer emitters. The employment of the multi-layer emitters is due to the fact that it is difficult to pole $>100-\mu \mathrm{m}$ thick polymer films with a voltage high enough to obtain good EO coefficients in our lab. ${ }^{11}$

Because of the transverse poling geometry, in addition to the operation on the LAPC emitter orientation we mentioned above, we also needed to rotate the LAPC sensor such that the angle between the incidence and the poling direction was $\sim 45^{\circ}$ leading to a projected component of the $p$-polarized $\mathrm{THz}$ field along this poling direction, and further needed to rotate the LAPC sensor until the incidence plane was $45^{\circ}$ with respect to the probe beam polarization for sensitive EO detection. These rotations of the LAPC sensor make the utilization of the transversely poled EO polymer possible, but the full strength of the incident $\mathrm{THz}$ field and nonlinearity of the EO polymer are not accessed. Instead, using in-plane poling would be a very attractive alternative for the sake of increasing the sensitivity of the EO polymer sensors as they can be oriented such that the poling direction is parallel to the incident $\mathrm{THz}$ field. We are currently pursuing this technique in our lab. 

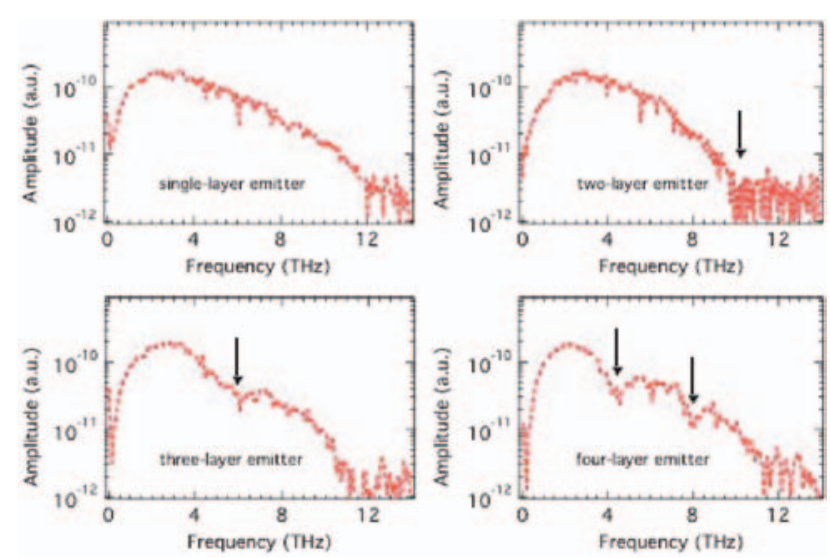

Fig. 6. Amplitude spectra of the THz fields from multi-layer LAPC emitters. The same LAPC sensor was used in the four cases. The spectral dips for the three- and four-layer emitters are due to phase-mismatching in LAPC.

Figure 6 shows the amplitude spectra of the THz fields from the four stacked LAPC emitters, using the same LAPC sensor in each case. A $\sim 12-\mathrm{THz}$ bandwidth free of any spectral gap is achieved for the single-layer emittersensor pair. For the two-layer LAPC emitter, the observable bandwidth is narrowed to $\sim 11 \mathrm{THz}$. It is very interesting to note that, for the three- and four-layer thick LAPC emitter, there are clear phase-mismatch induced spectral dips. For the former one, the dip is located around $5 \mathrm{THz}$, and for the latter one, one dip is located at $\sim 4 \mathrm{THz}$ and the other at $\sim 8 \mathrm{THz}$. Our modeling work (see Section 5) shows that these dips match our prediction.

Many water vapor absorption lines are observed for the four spectra, because the air of the experiments was not completely dry. The $\mathrm{THz}$ field peak-to-peak amplitudes in these four cases were quite comparable, leading to the conclusion that there is no need to use an LAPC emitter thicker than $200 \mu \mathrm{m}$ to achieve useful and smooth bandwidth to $\sim 12 \mathrm{THz}$.

Because ZnTe and some other EO crystals with similar crystallographic structure have been used as standard $\mathrm{THz}$ emitters and sensors, it is meaningful to compare the performance of our LAPC films with an EO crystal for emission efficiency or detection sensitivity. We had a $\sim 80$ $\mu \mathrm{m}$ thick $\mathrm{ZnCdTe}$ crystal available and used it as the $\mathrm{THz}$ sensor to compare with our LAPC sensor. Figure 7 shows the comparison of the amplitude spectrum between the ZnCdTe sensor and the LAPC sensor, where the same single layer LAPC emitter was used. For the $\mathrm{ZnCdTe}$ sensor (black line), there is a strong absorption gap around $5 \mathrm{THz}$, in contrast to the continuous spectrum from the LAPC emitter-sensor pair (red line). The locations of the water vapor absorption lines above $7 \mathrm{THz}$ are well overlapped for both spectra. The $\mathrm{ZnCdTe}$ sensor is about 4 times more sensitive than the LAPC sensor for lower frequencies around $2 \mathrm{THz}$, which is due to the fact that there is good phase-matching in $\mathrm{ZnCdTe}$ in that spectral region. On the

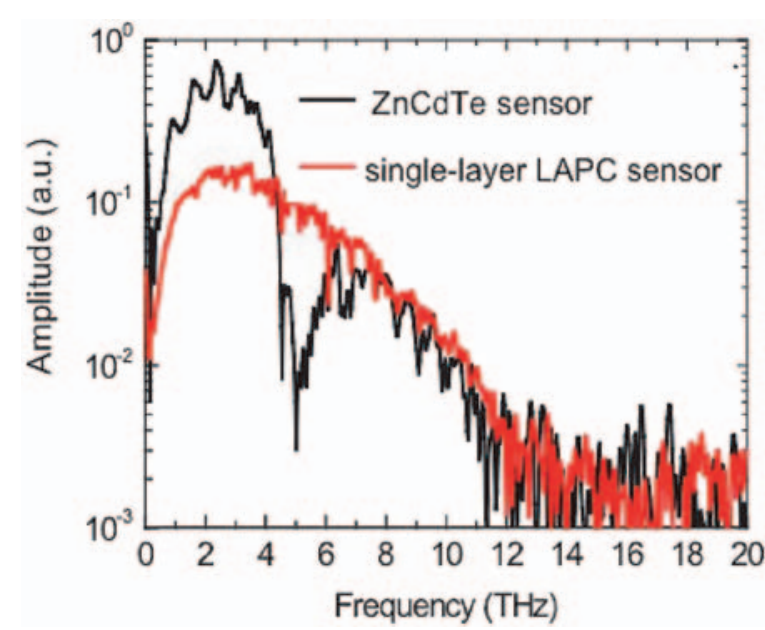

Fig. 7. Comparison of the $\mathrm{THz}$ spectra with an $80-\mu \mathrm{m}$ thick $\mathrm{ZnCdTe}$ sensor and a single-layer LAPC sensor. The same single-layer LAPC emitter is used in the two cases. The wide spectral gap in the case of the $\mathrm{ZnCdTe}$ sensor is due to phonon absorption associated with the lattice resonance of the crystal.

other hand, the phase-mismatch in LAPC is constant in the whole observable THz range $\left(n_{g} \approx 1.93, n_{\mathrm{THz}} \approx 1.71\right.$, see Section 4.5), and not as good as $\mathrm{ZnCdTe}$ around $2 \mathrm{THz}$, for the 800-nm probe wavelength. The other reason for the lower sensitivity of the LAPC sensor is due to the transverse poling geometry discussed above. Multiple internal reflections inside the $\sim 80-\mu \mathrm{m}$ thick $\mathrm{ZnCdTe}$ crystal explain the $\sim 0.7-\mathrm{THz}$-period spectral modulation. This effect is mostly eliminated in the case of the LAPC sensor due to the oblique incidence of the $\mathrm{THz}$ field and the lower refractive index of LAPC.

\subsection{DAPC Emitter and Multi-Layer LAPC Sensor Operated at $\sim 1300 \mathrm{~nm}$}

For field $\mathrm{THz}$ applications, portable systems are highly desired. As light sources are usually the most bulky part of THz systems, reduction of the size of the light sources is crucial. In this sense, femtosecond fiber lasers (usually emitting at $>1-\mu \mathrm{m}$-wavelength) are the most promising candidates. Indeed, some work has been done towards this goal. For all-optical $\mathrm{THz}$ systems with light sources emitting in the telecommunication wavelength bands, published work exists for $\mathrm{GaAs}^{68}$ and DAST. ${ }^{63}$ GaAs has a good phase-matching property in the telecommunication bands and its phonon resonance occurs at $\sim 8 \mathrm{THz}$ (quite high compared to ZnTe). It should be good for a broadband $\mathrm{THz}$ system whether used as a THz emitter or sensor, except that its EO coefficient is very small. This feature keeps one from building a bright $\mathrm{THz}$ system. On the other hand, while DAST has quite high EO coefficients in the telecommunication bands, the material is mechanically fragile and of high fabrication cost, not to mention the multiple phonon bands affecting the smoothness of the $\mathrm{THz}$ spectrum. Most conventional EO materials such 
as $\mathrm{ZnTe}$ and $\mathrm{GaP}$ do not have suitable properties in the telecommunication bands.

For the niche requirements associated with the use of light sources in the telecommunication bands, EO polymers may play a large role. Thanks to more than twenty years of intensive study driven by the telecommunication industry crying for cheap EO devices and easy integration, EO polymers with both high EO coefficients and high glass transition temperature (good thermal stability) are available now. ${ }^{69-71}$ With certain material modification, $\mathrm{THz}$ technology can also benefit from this advancement. An example to show the necessity of the material modification is that most existing NLO chromophores that lead to EO polymers with high EO coefficients cannot be processed into the thick films required by freestanding $\mathrm{THz}$ emitters or sensors. Of course, one can alternatively resort to a guided-wave device configuration ${ }^{72}$ to build $\mathrm{THz}$ emitters and sensors. In this case, polymer films can be thin (a few microns) but the interaction length should be relatively long (a few millimeters). Then, in addition to the obvious requirement of a good phase-matching property for the material, its group velocity dispersion (GVD) at the pump wavelength should be very small such that pulse duration can remain approximately the same all along the waveguide, and there should be high transparency in both the optical and $\mathrm{THz}$ regime (or, both the optical absorption and $\mathrm{THz}$ absorption in the waveguide should be low) too. It is challenging to squeeze all the required properties into a single material. Therefore, before reaching the point of building guided-wave $\mathrm{THz}$ emitters and sensors, material tailoring and characterization should first be done.

At this point, DAPC and LAPC are two mature EO composites ready for $\mathrm{THz}$ application. As shown above, they work quite well at the $800-\mathrm{nm}$-wavelength pump. Measurements of their refractive indices in both the optical and $\mathrm{THz}$ regime (see Section 4.5) show that in the wavelength tuning range $(1260 \mathrm{~nm}-1500 \mathrm{~nm})$ of typical optical parametric amplifiers (OPA), both materials have better phasematching than at $\sim 800 \mathrm{~nm}$. The shortcoming for the two materials, however, is that their EO coefficients in the OPA tuning range are not very high: $r_{33}<20 \mathrm{pm} / \mathrm{V}$. Nevertheless, we have used these two materials in $\mathrm{THz}$ systems with very low pump power $(<7 \mathrm{~mW})$.

In one experiment, we tuned the OPA to $\sim 1300 \mathrm{~nm}$, used a $\sim 75-\mu \mathrm{m}$-thick DAPC film as the $\mathrm{THz}$ emitter, and either a 2-mm-thick ZnTe crystal or a $80-\mu \mathrm{m}$-thick $\mathrm{ZnCdTe}$ crystal as the $\mathrm{THz}$ sensor. The measured timedomain $\mathrm{THz}$ waveforms and corresponding frequencydomain $\mathrm{THz}$ spectra are shown in Figure 8 . In both cases, $<7 \mathrm{~mW}$ (measured after the mechanical chopper) optical pump power illuminated the emitter. In addition to the clear demonstration of the capability of EO polymers of generating $\mathrm{THz}$ radiation with $\sim 1300$-nm-wavelength pump, we also observe significant phase-mismatching induced effect due to the use of ZnTe. It is known that $\mathrm{ZnTe}$ and $\mathrm{ZnCdTe}$ have very similar dielectric properties
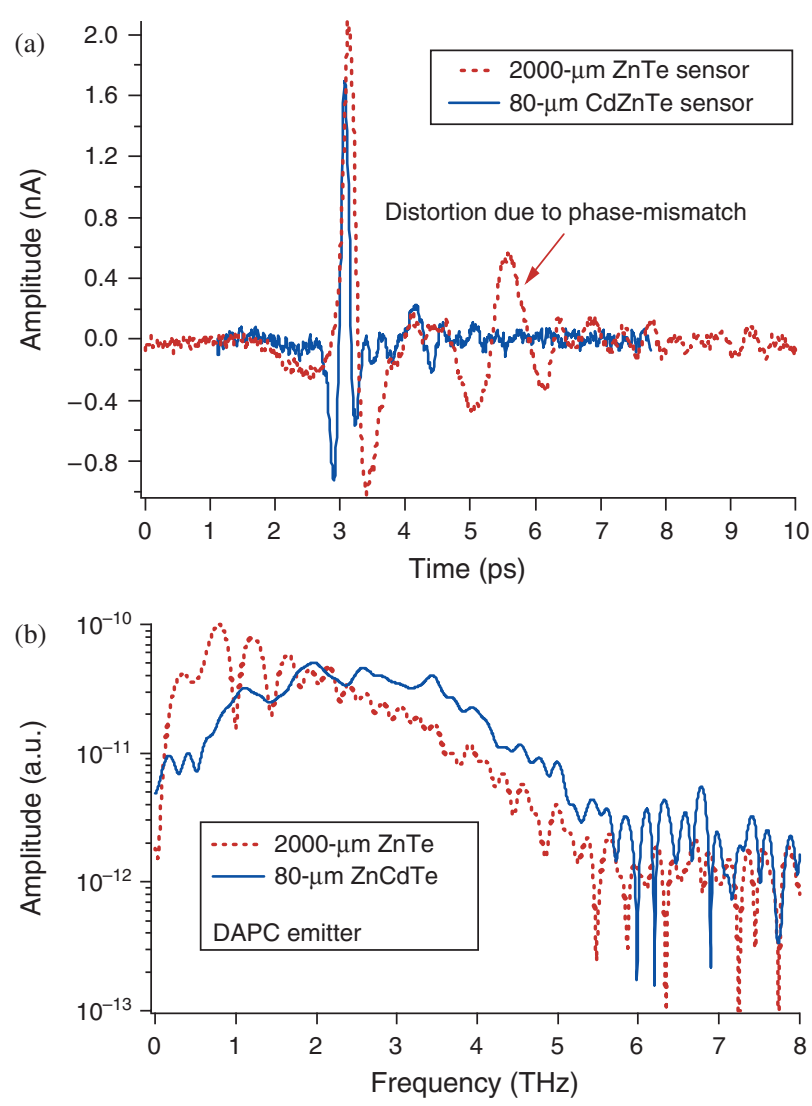

Fig. 8. THz amplitude spectra obtained from a DAPC emitter$\mathrm{ZnTe} / \mathrm{ZnCdTe}$ sensor pair operated at $\sim 1300 \mathrm{~nm}$. Spectral dips corresponding to the ZnTe sensor are due to the phase mismatching in the crystal, while the oscillations corresponding to the $\mathrm{ZnCdTe}$ sensor are due to the multiple reflections in the $80-\mu \mathrm{m}$ crystal.

and EO coefficients. From Figure 8, however, we see that the much thicker $\mathrm{ZnTe}$ did not provide much larger $\mathrm{THz}$ signal than the $\mathrm{ZnCdTe}$. This can only be explained by the significant phase-mismatching and/or THz absorption condition in the $\mathrm{ZnTe}$. The spectrum associated with the $\mathrm{ZnTe}$ clearly shows many phase-mismatching induced spectral dips. It should be noted that the oscillations on the top of the spectrum corresponding to the thin ZnCdTe sensor are due to the multiple reflections in the crystal, confirmed by the oscillation period and the crystal thickness. As will be described in Section 5, our modeling work can exactly predict the results shown here. This experiment clearly shows the importance of good phase-matching in optical $\mathrm{THz}$ generation and detection systems. In a recent study on the phase-matching in OR, van der Valk et al. ${ }^{73}$ also observed spectral dips from ZnTe emitters using an near-infrared optical parametric oscillator and successfully modeled these dips.

Because of the existing phonon absorption at $\sim 5.3 \mathrm{THz}$ in $\mathrm{ZnCdTe}$, even for the $80-\mu \mathrm{m}$-thick $\mathrm{ZnCdTe}$, we could not observe bandwidth broader than $5 \mathrm{THz}$. We wanted to check the bandwidth performance of the EO polymers. Therefore, we used a $60-\mu \mathrm{m}$-thick $c$-cut DAST crystal as the $\mathrm{THz}$ emitter, since DAST has quite high EO 


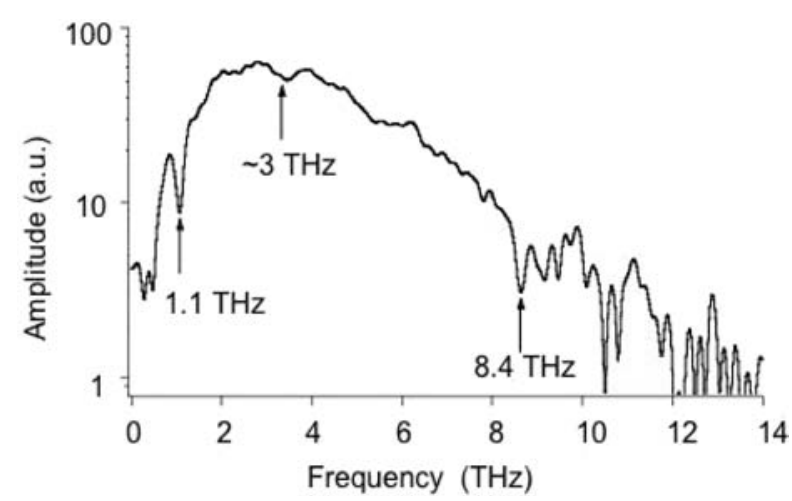

Fig. 9. Broadband $\mathrm{THz}$ spectrum obtained from the DAST emitterLAPC sensor pair operated at $\sim 1300 \mathrm{~nm}$. The dips pointed out by the arrows are attributed to the multiple phonon absorption bands in DAST.

coefficients at the wavelength and the $\mathrm{THz}$ absorption due to its multiple phonon bands is expected to be small (because of the weak intermolecular interaction) in the thin DAST. LAPC was used as the THz sensor in this case. The experimental results are shown in Figure 9. Clearly, $>10$ $\mathrm{THz}$ bandwidth is possible with this emitter-sensor combination. Because of the smooth and broadband response of the EO polymers, we can confidently conclude that the spectral dips seen on the spectrum are actually due to the DAST lattice resonances. In Section 3.4, we mentioned that IR spectroscopy is not suitable to identify weak absorptions associated with the lattice vibration of DAST. In our $\mathrm{THz}$ experiment, they are easily identified.

With LAPC having good phase-matching property at $\sim 1300 \mathrm{~nm}$, it was interesting to find out how far we can go by simply increasing the interaction length. Therefore, we conducted experiments on multi-layer LAPC emitters (see Fig. 10) at this wavelength. Surprisingly, the

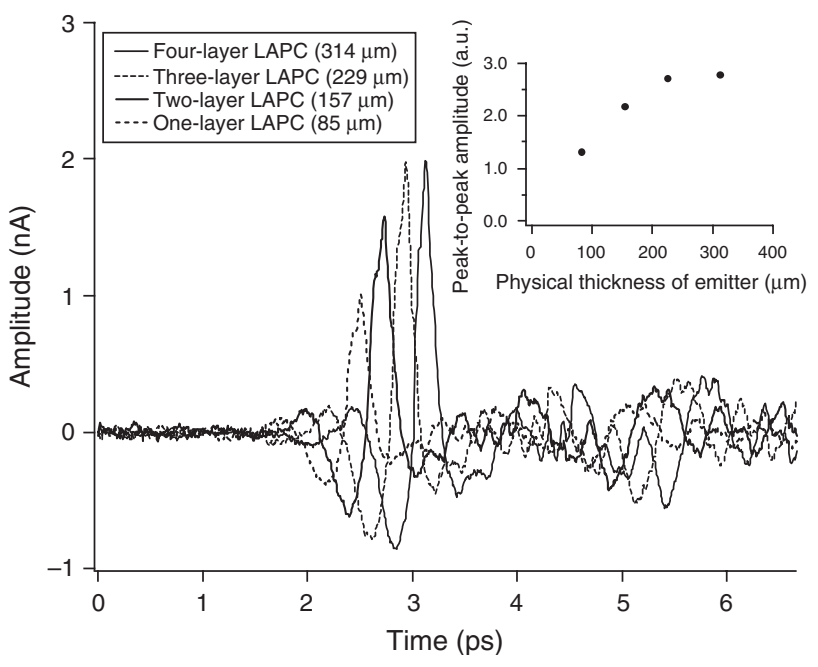

Fig. 10. THz waveforms obtained from multi-layer LAPC emitters, measured by a 2-mm ZnTe sensor. All the emitters and the sensor were operated at $\sim 1300 \mathrm{~nm}$. The inset shows the peak-to-peak amplitude versus the physical thickness of the emitters. four-layer LAPC emitter did not provide more $\mathrm{THz}$ radiation than the three-layer LAPC emitter. This does not agree with our expectation out of the phase-matching study (see Section 4.5). Tentatively, we think that the disagreement might be due to the $\mathrm{THz}$ absorption in LAPC, which we neglected in our theoretical study. It becomes crucial at this point that we understand this issue if we want to make relatively long guided-wave $\mathrm{THz}$ emitters.

\subsection{DAST Emitter}

Concern about multiple $\mathrm{THz}$ absorption bands of DAST has always existed. While an absorption band centered at $\sim 1.1 \mathrm{THz}$ has long been identified from the THz-TDS study $^{59}$ and some THz emission experiments involved with DAST emitters, ${ }^{54,63}$ consistency is yet to come concerning the absorption bands at higher frequencies. ${ }^{63,74}$ As shown above, the $\mathrm{THz}$ emission experiment from the thin DAST crystal shows higher sensitivity to $\mathrm{THz}$ absorption than the infrared spectroscopy in the lower frequency end in the FIR regime $(<12 \mathrm{THz})$. With the help of the broadband LAPC sensor, we made a detailed investigation of the use of DAST as a broadband THz emitter.

Our $c$-cut DAST crystal was purchased from Rainbow Photonics. ${ }^{75}$ For this specific cutting, only two elements of the $\chi^{\mathrm{OR}}$ tensor- $\chi_{111}^{\mathrm{OR}}$ and $\chi_{122}^{\mathrm{OR}}$ can be accessed for OR. Other elements are either too small or cannot be accessed by the pump beam (with normal incidence) polarized parallel to the $a b$ plane. For an $a$-polarized pump beam, $\chi_{111}^{\mathrm{OR}}$ is involved in optical rectification; while for a $b$-polarized pump beam, $\chi_{122}^{\mathrm{OR}}$ is involved. In both cases, the generated THz is $a$-polarized, which can easily be identified by calculating the pump-induced nonlinear polarization for OR. $\chi_{111}^{\mathrm{OR}}(1230 \pm 130 \mathrm{pm} / \mathrm{V})$ is almost an order of magnitude higher than $\chi_{122}^{\mathrm{OR}}(166 \pm 16 \mathrm{pm} / \mathrm{V})$ for DAST at $800 \mathrm{~nm} .{ }^{63}$ To take advantage of the large $\chi_{111}^{\mathrm{OR}}$, it seems that a DAST crystal should be oriented such that the pump beam is $a$ polarized for $\mathrm{THz}$ emission. This arrangement has actually been taken by most researchers. The issue here is that good phase-matching does not exist in the small wavelengthtuning range of a Ti:sapphire laser. Therefore, while $\chi_{111}^{\mathrm{OR}}$ is large, the phase-mismatching reduces the $\mathrm{THz}$ emission efficiency at the same time.

As pointed out in Ref. [63], using a $b$-polarized pump should result in better phase-matching in the tuning range of a Ti:sapphire laser system, while for the tuning range of a NIR OPA, $a$-polarized pump is preferred. Probably because of a lack of a thin crystal, the authors did not provide experimental results. Using our thin crystal, we should be able to see the difference. With a chirped pulse amplifier system based on Ti:sapphire (SpectraPhysics Spitfire, $800 \mathrm{~nm}, 50 \mathrm{fs}, 1 \mathrm{kHz}$ ) and an optical parametric amplifier (SpectraPhysics OPA, tuned at $1300 \mathrm{~nm}$, $<100$-fs-duration, $1 \mathrm{kHz}$ ) as light sources, respectively, we studied the polarization dependence of the phase-matching versus wavelength. 


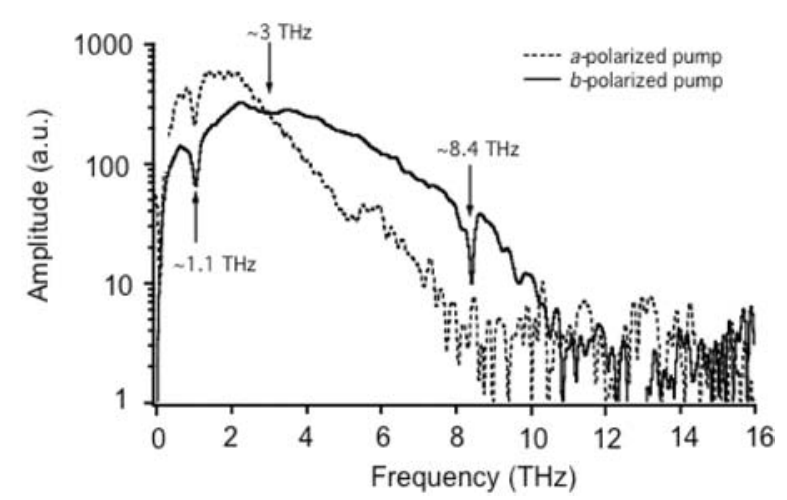

Fig. 11. THz amplitude spectra obtained from a DAST emitter-LAPC sensor pair operated at $800-\mathrm{nm}$ wavelength with $a$ - and $b$-polarization pump, respectively.

In the case of the use of the Spitfire, the DAST emitter was carefully placed away from the focal point of the parabolic mirror to avoid the $\sim 70 \mathrm{~mW}$ chopped pump power from damaging the crystal. For this arrangement, the size of the pump is quite large $\left(\sim 5 \mathrm{~mm}^{2}\right)$. In the case of the use of the OPA, the DAST emitter was placed at the focal point in order to make full use of the average $<7$-mW (maximal power available) chopped power. The beam size at the focal point was smaller than $1 \mathrm{~mm}^{2}$. To align the pump beam polarization parallel either to the $a$ axis or to the $b$-axis of DAST, we used a half-wave plate in front of the DAST crystal. The generated $\mathrm{THz}$ field was collimated and focused onto the LAPC polymer sensor by four off-axis parabolic mirrors.

Figure 11 shows the $\mathrm{THz}$ amplitude spectra from the DAST emitter-LAPC sensor pair when the 800-nm pump was $a$ - and $b$-polarized, respectively. Clearly, under the same pump power, the high-frequency components of $\mathrm{THz}$ emission from the $a$-polarized pump drop faster than from the $b$-polarized pump. According to our modeling work for OR and EO sampling (as described below in Section 5), this can be expected from stronger dispersion and/or worse phase-matching for the $a$-polarized pump. Because the absorption peak for $a$-polarized light is at $\sim 530 \mathrm{~nm}$, being longer than $\sim 500 \mathrm{~nm}$ for $b$-polarized light, the former optical polarization exhibits stronger dispersion at $800 \mathrm{~nm}$. To evaluate the phase-matching condition, we need knowledge not only of optical group indices but also of the $\mathrm{THz}$ indices of DAST. Previous work has provided the optical refractive indices of DAST, allowing us to do the calculation for its optical group indices. At $800 \mathrm{~nm}$, optical group indices of $\sim 3.38$ and $\sim 1.98$, have been obtained from the calculation for the $a$ - and $b$-polarized beam, respectively. ${ }^{56}$ However, the published work on the measurements of absorption coefficients and refractive indices of DAST in the $\mathrm{THz}$ regime provided results only up to $\sim 3 \mathrm{THz},{ }^{59}$ which is much narrower than the bandwidth we observed in this work. Around the $\sim 1.1-\mathrm{THz}$ phonon absorption band, the refractive index was found to have a dramatic change from 1.6 to 3.2. At the upper limit of this bandwidth, the $\mathrm{THz}$ indices tend to be a constant of $\sim 2.3$. If we assume that above $3 \mathrm{THz}$ the $\mathrm{THz}$ indices are still $\sim 2.3$, then a $b$-polarized pump clearly provides better phase-matching for OR than an $a$-polarized pump. Accordingly, we should expect more efficient emission of high-frequency $\mathrm{THz}$ components from a $b$-polarized pump. This is exactly what we observe from Figure 11: the spectrum corresponding to a $b$-polarized pump exhibits higher amplitudes above $3 \mathrm{THz}$. The overall observable amplitudes are comparable for both cases (with the same noise level), even with the fact that $\chi_{111}^{\mathrm{OR}}$, related to an $a$ polarized pump, is almost an order of magnitude higher than $\chi_{122}^{\mathrm{OR}}$.

Both spectra clearly show a sharp and narrow opticalphonon resonance around $1.1 \mathrm{THz}$, as expected. Another absorption gap centered at $\sim 3 \mathrm{THz}$, however, is much broader and shallower, and can be clearly seen only from the spectrum corresponding to the $b$-polarized pump. The lack of this absorption band in the case of the $a$-polarized pump might be due to a sharp reduction of spectral amplitudes for frequencies above $\sim 2 \mathrm{THz}$, as this reduction might eliminate the shallow and broad absorption. Thanks to the broad measurement bandwidth of our LAPC sensor, we can also identify a sharp and narrow resonance at $\sim 8.4$ $\mathrm{THz}$, which is very close to the previously reported 8.5 THz absorption of DAST by Taniuchi et al. ${ }^{74}$ who used DFG method to generate $\mathrm{THz}$ radiation. To understand the origination of the multiple resonances in organic crystals, rigid modeling work is needed.

In contrast with the 800-nm-wavelength pump, the use of a $1300-\mathrm{nm}$-wavelength pump favors the $a$-polarization instead of $b$-polarization. This is due to the optical group index of 2.39 for an $a$-polarized pump being much closer to the $\mathrm{THz}$ index of $\sim 2.3$ for high $\mathrm{THz}$ frequencies than that of 1.67 for $b$-polarized pump at this wavelength ${ }^{56,59}$ We actually cannot observe $\mathrm{THz}$ generation from the $b$ polarized pump at this wavelength. We attribute this to the smaller pump power $(<7 \mathrm{~mW})$, wider pulse duration, and the much smaller NLO coefficient $\chi_{122}^{\mathrm{OR}}(1300 \mathrm{~nm}$ is far away from resonance enhanced region for nonlinear processes) in this case. On the other hand, for the $a$-polarized pump, a $\sim 10$-THz-bandwidth emission is obtained even with a much smaller $\chi_{111}^{\mathrm{OR}}$ (compared with the case of an 800-nm-pump) at this wavelength. This can only be explained by good phase-matching in this case. The amplitude spectrum also exhibits $\sim 1.1-\mathrm{THz}, \sim 3-\mathrm{THz}$, and $\sim 8.4$ $\mathrm{THz}$ resonances (see Fig. 9), just as in the case of the 800-nm-wavelength pump.

As seen from our experimental results, $\mathrm{THz}$ absorption in the multiple bands of our thin DAST crystal is not very strong, in comparison with inorganic EO crystals (see Fig. 7 , where the $80-\mu \mathrm{m} \mathrm{ZnCd}$ sensor caused a wide and strong absorption centered at $\sim 5.3 \mathrm{THz}$ ). Thus, for a thin DAST crystal, a mostly continuous broad bandwidth can be achieved. If the cost of fabrication can be reduced (especially considering the fact that the material is mechanically 

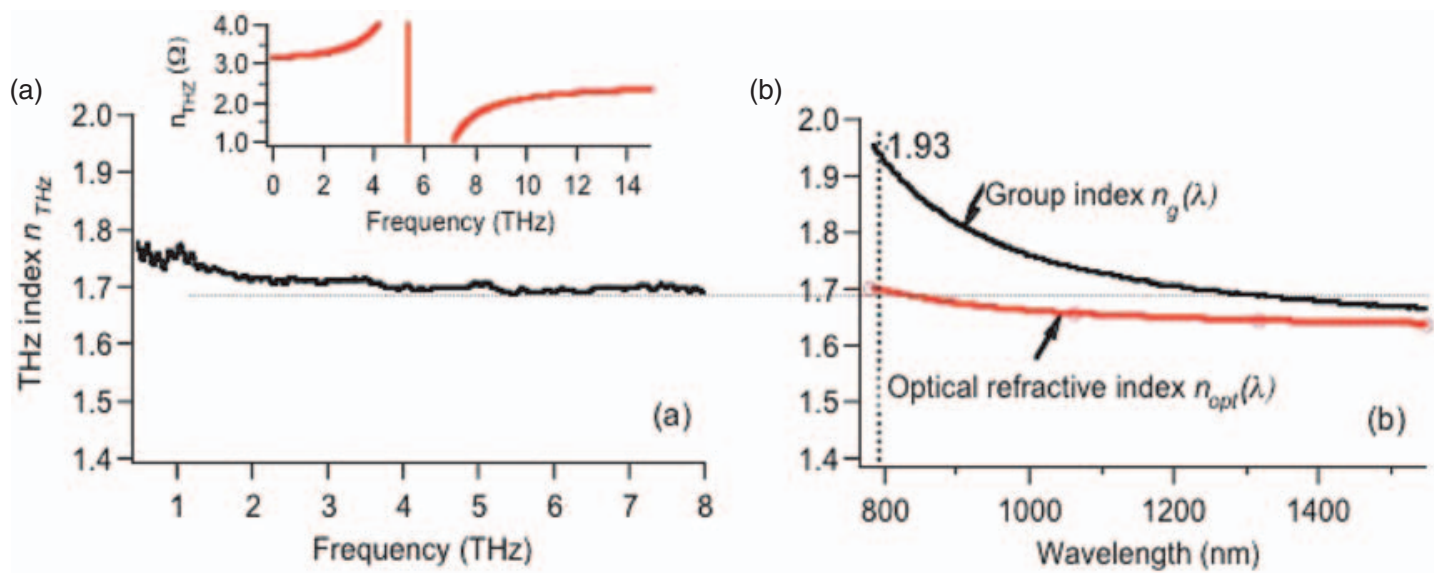

Fig. 12. (a) Refractive indices of LAPC in the THz regime, measured using THz-TDS. The inset shows the refractive indices of ZnTe in the THz regime. (b) Derived refractive indices (red line) and group indices (black line) of LAPC in the optical regime. Open circles are measured refractive indices of LAPC at $780 \mathrm{~nm}, 1064 \mathrm{~nm}, 1319 \mathrm{~nm}$, and $1550 \mathrm{~nm}$, respectively.

and thermally fragile), DAST should be a very promising material for $\mathrm{THz}$ applications.

\subsection{Dielectric Property Characterization of EO Polymers Using THz-TDS}

Characterization of EO polymers in both the $\mathrm{THz}$ and optical regime is essential with regard to their THz applications. The optical characterization that includes EO coefficient, refractive index, and optical absorption can be easily done through established technology. In the $\mathrm{THz}$ regime, we employed the recently developed THz-TDS technique. Its operating principle has been explained in Section 2.4.

Figure 12(a) shows the measured $\mathrm{THz}$ indices for LAPC up to $\sim 8 \mathrm{THz}$ (below $0.5 \mathrm{THz}$ and above $8 \mathrm{THz}$, the SNR issue does not allow us to trust the results). So far, most THz-TDS systems only provide up to $\sim 3 \mathrm{THz}$ spectral coverage, which is far lower than our system. From Figure 12(a), we see that the THz indices of LAPC are approximately constant in the whole range of spectral coverage. This feature of constant refractive indices in the $\mathrm{THz}$ regime is general for all amorphous EO polymers as there exists no lattice structure.
In contrast, crystalline materials exhibit dramatic changes of the $\mathrm{THz}$ indices around their lattice resonance. The inset of Figure 12(a) shows the $\mathrm{THz}$ index of $\mathrm{ZnTe}$, calculated from an oscillator model. The dispersion curves of $\mathrm{ZnTe}$ suggest that it is impossible to achieve good phase-matching for a broad $\mathrm{THz}$ region in crystalline materials. For ZnTe, good phase-matching is only exhibited at $\sim 2-\mathrm{THz}$ with an 800 -nm-wavelength pump. In the case of EO polymers, once a suitable pump wavelength is found, the whole region of $\mathrm{THz}$ radiation enjoys good phase matching. Therefore, a broad spectral-gap free $\mathrm{THz}$ spectrum is possible.

Figure 12(b) shows the refractive indices $n_{\text {opt }}$ and group index $n_{\mathrm{g}}$ of LAPC obtained by measuring $n_{\text {opt }}$ at $780 \mathrm{~nm}$, $1064 \mathrm{~nm}, 1319 \mathrm{~nm}$, and $1550 \mathrm{~nm}$, respectively and then fitting the data to a Sellmeier dispersion formula (to obtain the dispersion of $n_{\mathrm{opt}}$ ) and performing numerical calculation:

$$
n_{\mathrm{g}}=n_{\mathrm{opt}}-\left.\lambda_{\mathrm{opt}} \cdot \frac{d n_{\mathrm{opt}}}{d \lambda_{\mathrm{opt}}}\right|_{\lambda_{\mathrm{opt}}}
$$

(to obtain the dispersion of $n_{\mathrm{g}}$ ). As shown in Figure 12, for LAPC $n_{\mathrm{g}}(800 \mathrm{~nm}) \approx 1.93$ and $n_{\mathrm{THz}} \approx 1.71$, suggesting phase-mismatching. Nevertheless, we could still achieve
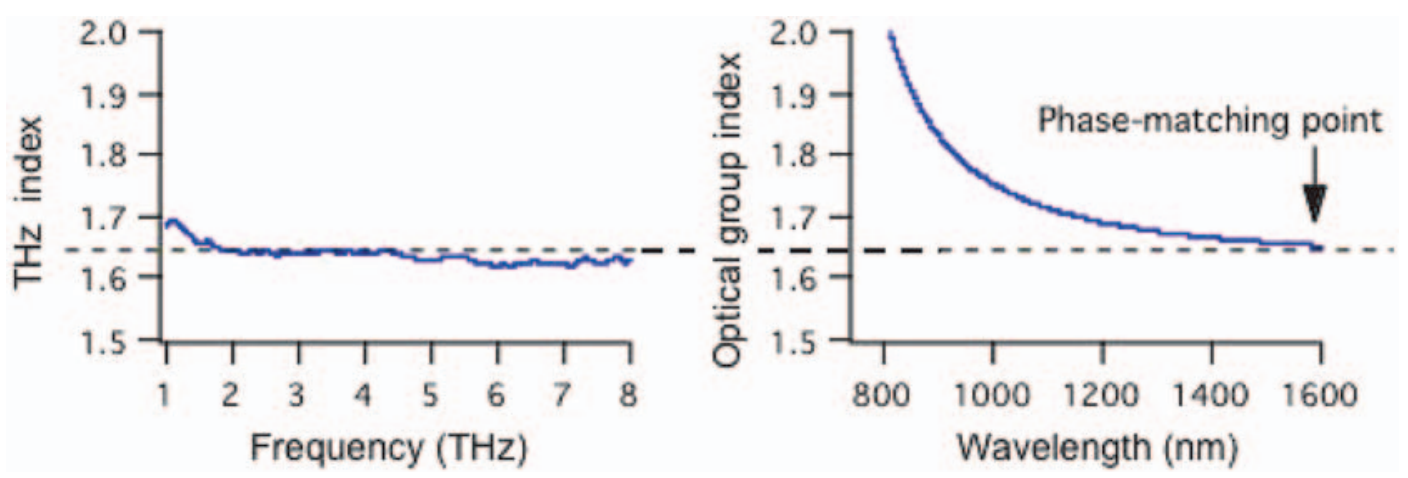

Fig. 13. Comparison of the THz indices (left) with the optical group indices (right) of DAPC shows that the phase-matching point is out of the wavelength tuning range of the OPA. 
$\sim 12-\mathrm{THz}$ bandwidth for LAPC emitter sensor pair. It should be noted that at longer wavelengths $(>1300 \mathrm{~nm})$, even better phase-matching exhibits for LAPC.

Using the same method on DAPC, we evaluated its phase-matching property, as shown in Figure 13. At 800 $\mathrm{nm}$, the phase-mismatching for DAPC is worse compared with LAPC. Better phase-matching can be obtained in the OPA wavelength tuning range, but unlike LAPC, there is no perfect phase-matching in the range.

An additional problem is that both LAPC and DAPC have a relatively small EO coefficient in this wavelength regime. In addition, our THz-TDS study also found that $\mathrm{THz}$ absorption for these composites was high across the useful bandwidth (although there is no distinguished absorption fingerprint). Accurate absorption coefficients are yet to be determined, depending on further SNR improvement. Tentatively, we think that the $\mathrm{THz}$ absorption is what keeps us from using a long interaction-length for LAPC to achieve higher conversion efficiency in the $\mathrm{THz}$ generation and higher sensitivity in the $\mathrm{THz}$ detection when the wavelength-tunable OPA is used (see Fig. 7).

\section{MODELING A POLYMER EMITTER-SENSOR PAIR}

Many factors, such as laser pulse duration and bandwidth, dielectric properties of an EO material, and phase-matching condition, affect the $\mathrm{THz}$ generation and detection severely. From the viewpoint of system design, it is useful to be able to predict the performance of a $\mathrm{THz}$ system. A suitable model is needed towards this goal. Especially, seeking/designing the best EO polymer from a wide variety of material possibilities requires the help of reliable modeling work.

The generation of the THz radiation via OR is governed by Eq. (1) (in Section 2.2), the frequency-domain version of the nonlinear wave-mixing process. The analytical solution of this equation was derived by Faure et al. ${ }^{30}$ assuming that the pump pulse is transform-limited and remains the same all the way inside the EO medium (i.e., there is no optical absorption and dispersion for the EO medium) and that the dielectric constant $\varepsilon(\Omega)$ is not dependent on $\mathrm{THz}$ frequency. An analytical solution for the time-domain version of the nonlinear wave-mixing equation, was given even earlier by $\mathrm{Xu}$ et al., ${ }^{76}$ with the similar assumptions. In fact, $\varepsilon(\Omega)$ is far from being a constant for EO crystals and the optical dispersion indeed cannot be ignored. Recently, Schneider et al. gave a more completed solution to Eq. (1), where $\varepsilon(\Omega)$ can be complex (indicating an existence of $\mathrm{THz}$ absorption). ${ }^{63}$ Going one step further, we would like to add a complicated laser pulse profile and the complication associated with the optical pulse propagation effects inside the EO medium into consideration. The real situation we see in our experiments is the reason behind these considerations: the short laser pulses with a broad bandwidth are usually not transform-limited, and the central wavelength of the optical pump is usually located at the tail of the EO polymers' absorption band such that the GVD effect broadens the pulse duration quickly. In this case, an analytical solution to Eq. (1) is not possible. Therefore, we chose to numerically solve Eq. (1). The full work was presented elsewhere. ${ }^{77}$

As for the detection, a transfer function needs to be derived. In Section 2.3, we gave the expression for the $\mathrm{THz}$ signal measured by the EO detection. There we followed the physical picture of a traveling-wave EO modulator. Gallot et al..$^{33}$ have provided another picture of the EO detection, where nonlinear wave-mixing, instead of an EO modulator model, is used to derive the transfer function. The two models can be proved to be equivalent. In Ref. [77], we modified Gallot's model simply by adding the effects of the complicated probe pulse profile and the pulse distortion during propagation.

In each of our simulations, the obtained $\mathrm{THz}$ spectrum is the generated $\mathrm{THz}$ spectrum from the emitter multiplied by the transfer function of the detector. We categorized our simulations into two groups: the effect of the incident pulse profile complexity and the effect of the EO medium dielectric properties. We will briefly give the conclusions in this review. Interested readers can see Ref. [77] for details.

First, let us take a look at the effect of the incident pulse. We found when a very short laser pulse was involved, not only the bandwidth of the pulse but also its initial profile affected the obtained $\mathrm{THz}$ spectrum. For example, a transform-limited 10-fs (FWHM) Gaussian pulse has a 90-nm bandwidth. If another pulse has the same bandwidth and a third-order-dispersion (TOD) of $1000 \mathrm{fs}^{3}$ (not transform-limited any more), the pulse will be broadened to $\sim 19$ fs. When the two types of pulses are used with an EO polymer $\left(n_{\mathrm{g}}=1.93, n_{\mathrm{THz}}=1.73\right)$ emitter-sensor pair, our simulation (see Fig. 14) shows that the transformlimited pulse gives us a flatter and broader $\mathrm{THz}$ spectrum (also corresponds to more brightness). On the other hand, Figure 14 also shows two cases where a narrow bandwidth is involved. Here the $\mathrm{THz}$ spectra are exactly the same with or without the $1000 \mathrm{fs}^{3}$ TOD in the laser pulses. This simulation tells us that we should be aware of the importance of not only the laser bandwidth but also the pulse duration when we align a $\mathrm{THz}$ system involved with very short pulses to achieve a broadband and bright $\mathrm{THz}$ system.

In the second experiment we studied the effect of phasematching. As already seen in Sections 2.2 and 2.3, phasematching is a critical issue that not only affects system brightness and bandwidth but also determines the interaction length. Our simulation makes this much clearer. Between the two hypothetical EO polymers (see Fig. 15), EO polymer 2 has a better phase-matching property than the EO polymer 1 . As expected, for the same emitter thickness (the sensor thickness is fixed for all simulations in 


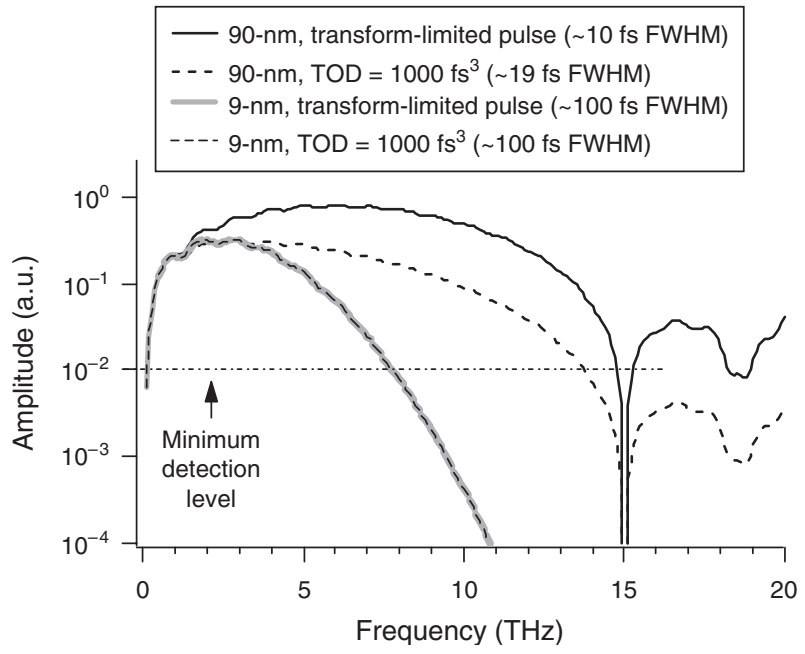

Fig. 14. Simulated $\mathrm{THz}$ amplitude spectra corresponding to optical pulses with different bandwidth and TOD. In the case that the optical pulses have broader bandwidth, TOD plays a large role with regard to both the $\mathrm{THz}$ bandwidth and brightness.

this experiment) EO polymer 2 provides much broader and brighter $\mathrm{THz}$ radiation. The performance can be further improved by increasing the interaction length of the EO polymer 2, while doing so for the EO polymer 1 only makes the performance worse as the brightness is barely improved and many phase-mismatch induced spectral dips appear.

In the third simulation, we studied the GVD effect inside EO materials. This effect is usually neglected by most published work. Through our simulation, we found that this effect was quite serious for very short laser pulses. In Figure 16, we can see that including GVD causes the bandwidth and brightness of the $\mathrm{THz}$ radiation to reduce significantly. On the other hand, when the pulse duration is long, the GVD effect does not play a large role. For a shorter pulse associated with a broader bandwidth, the

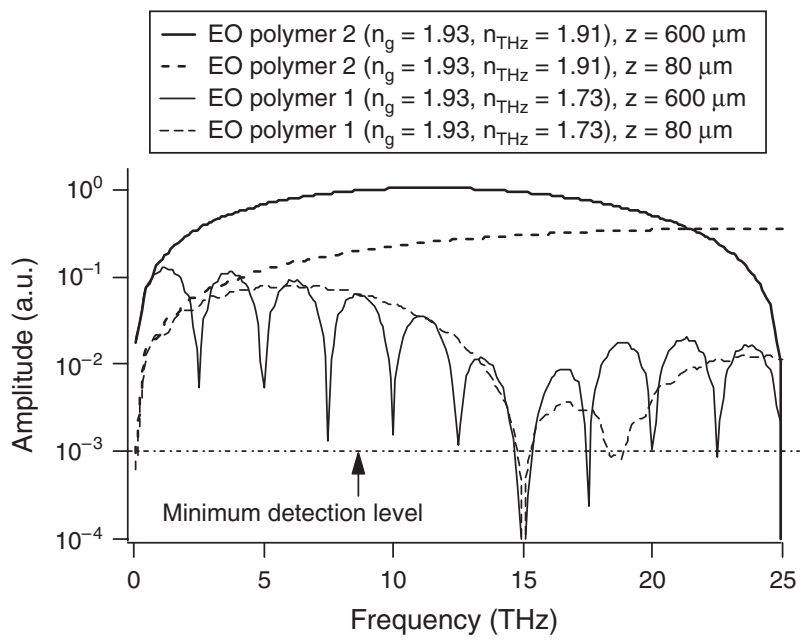

Fig. 15. Comparison of simulated $\mathrm{THz}$ amplitude spectra for emitters made of EO polymers with different phase-matching condition and thickness.

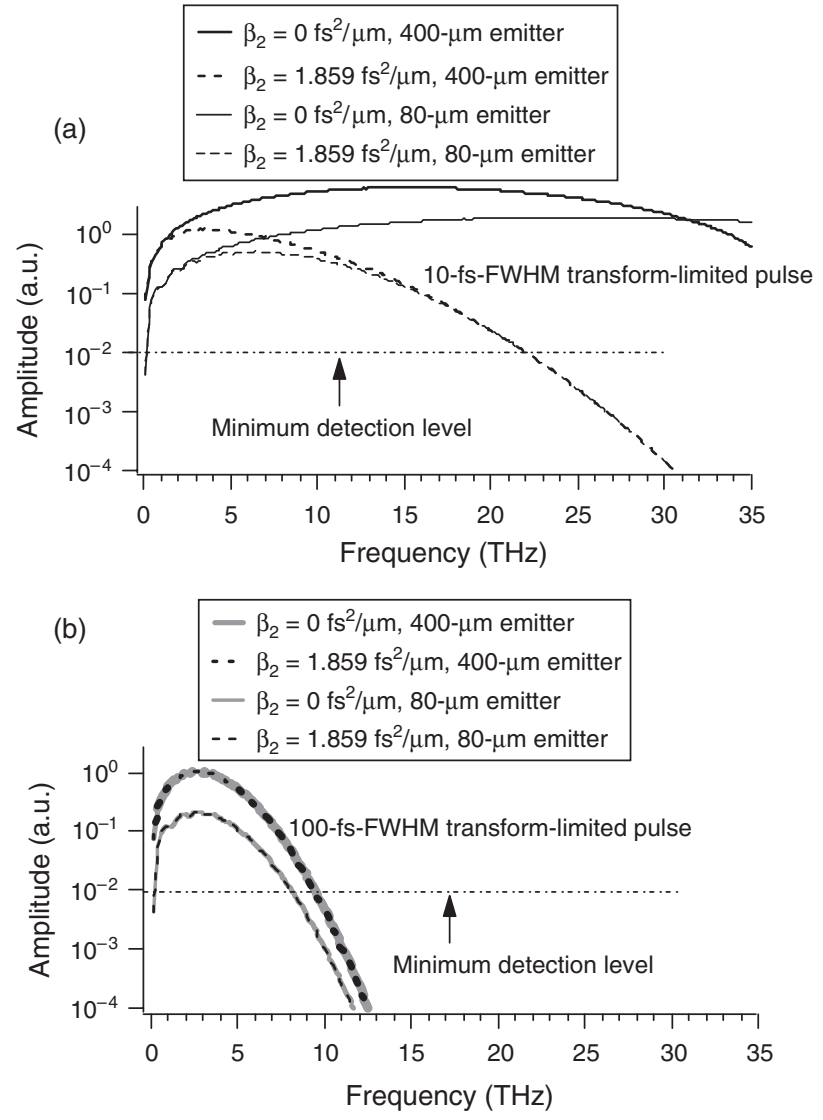

Fig. 16. GVD effect on the performance of the emitter-sensor pairs.

existence of the GVD effect in a medium broadens the pulse more seriously, leading to a worse $\mathrm{THz}$ bandwidth and brightness.

Finally, we checked the reliability of our model by comparing the model with experimental results. In one case, we wanted to predict the performance of the multilayer emitter-sensor pairs with the 800-nm-wavelength light source (the experimental results have been shown in Section 4.1). By simply varying the emitter thickness in the simulation with regard to the corresponding multi-layer
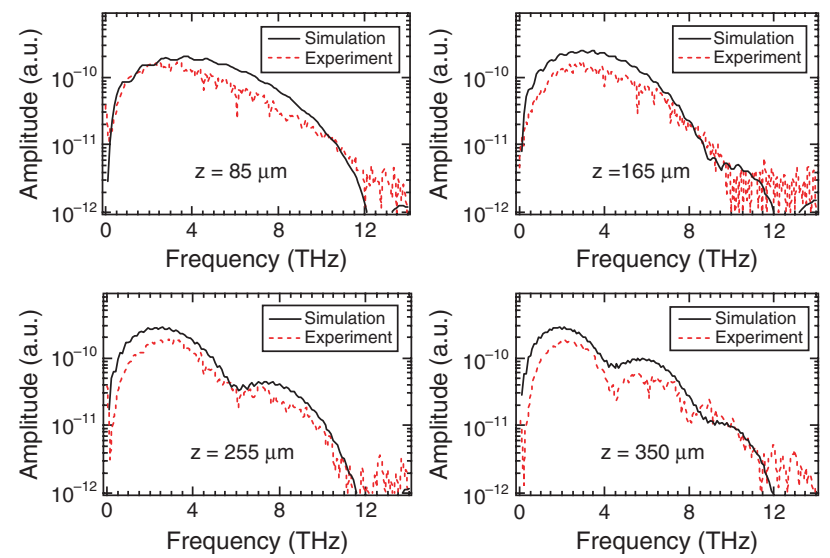

Fig. 17. Comparison of the simulation work (solid lines) with experimental results (dashed lines). 


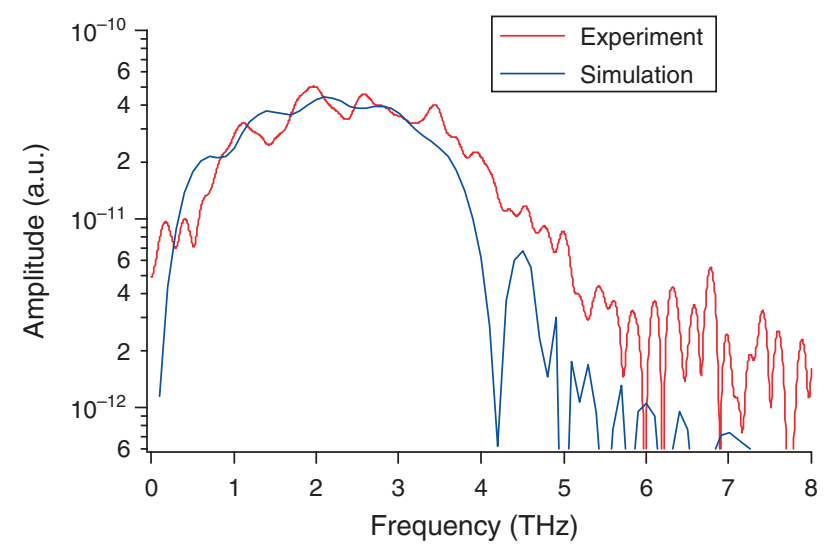

Fig. 18. Comparison of the simulation and the experiment for the DAPC emitter-ZnCdTe sensor pair operated at $\sim 1300 \mathrm{~nm}$.

LAPC emitter and using actual laser pulse characterization results and optical and $\mathrm{THz}$ index measurement results (see Section 4.4) for the other simulation parameters, we obtained simulated spectra nearly the same as the experiments. The comparison is shown in Figure 17.

In another case, we simulated the experiment described in Section 4.2, where the DAPC emitter-ZnTe/ZnCdTe sensor pairs were operated at $\sim 1300$-nm wavelength. Simulation parameters for the optical group index and $\mathrm{THz}$ index of DAPC were obtained from our measurements presented in Section 4.4. For ZnTe, we used the refractive index model presented by Gallot et al. ${ }^{32}$ A laser pulse duration of $\sim 100$ fs FWHM (quite wide, thus the TOD of the incident pulse and GVD of the materials can be neglected) was used, which was accurate within our pulse duration characterization error range. In the case of the 80- $\mu \mathrm{m} \mathrm{ZnCdTe}$ sensor, the $\mathrm{THz}$ spectrum from the simulation (solid black line of Fig. 18) is smooth up to $3.5 \mathrm{THz}$, which is consistent with the experiment (gray dotted line of Fig. 18). Since we did not include the effect of reflection in our model, it is not surprising that our simulation result does not show the spectral modulation that is exhibited in the experimental result. For the discrepancy that

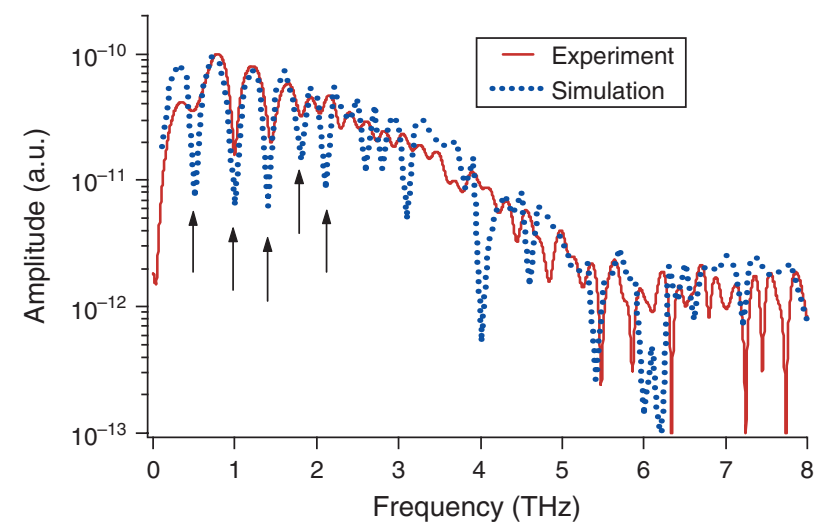

Fig. 19. Comparison of the simulation and the experiment for the DAPC emitter-ZnTe sensor pair, operated at $\sim 1300 \mathrm{~nm}$. occurs above $4 \mathrm{THz}$, we blame the lack of a model that can accurately predict the $\mathrm{THz}$ refractive indices around the lattice resonance $(\sim 5.3 \mathrm{THz}$ for $\mathrm{ZnTe})$. As for the 2$\mathrm{mm} \mathrm{ZnTe}$ sensor (Fig. 19), the simulation shows phasemismatch induced spectral dips, the first a few dips line up well with the experimental result. Like the $80-\mu \mathrm{m} \mathrm{ZnCdTe}$ sensor, most of the discrepancy between the experiment and simulation occurs around the lattice resonance range.

These two examples demonstrate the validity of our model. This model serves as a reliable guide for us to select new EO polymers in the future.

\section{CONCLUSIONS AND FUTURE WORK}

We have reviewed the application of organic materials as broadband $\mathrm{THz}$ emitters and sensors. Most of the discussion centered on organic EO materials including amorphous EO polymers such as LAPC and DAPC and organic salts such as DAST. For these materials, OR and EO sampling are the mechanisms for $\mathrm{THz}$ emission and $\mathrm{THz}$ detection respectively, and both processes strongly depend on high nonlinear coefficients. All the reviewed organic EO materials exhibit much higher nonlinear coefficients than their inorganic counterparts, with DAST providing the highest nonlinear coefficients at the most commonly available 800-nm wavelength (from Ti:sapphire lasers). When a broadband $\mathrm{THz}$ system free of any kind of spectral gaps is needed, however, amorphous EO polymers are advantageous over any other EO materials because of the intrinsic absence of the phonon absorption. We have experimentally demonstrated a spectral-gap free $\mathrm{THz}$ system with a useful bandwidth of $\sim 12 \mathrm{THz}$ based on a LAPC emittersensor pair. Using this system, we were able to conduct THz-TDS studies to characterize dielectric properties of materials. The $\mathrm{THz}$ indices of DAPC and LAPC were obtained in this manner and used to evaluate their phasematching properties in OR and EO sampling. We found that both LAPC and DAPC had approximately flat $\mathrm{THz}$ indices across a wide bandwidth (up to at least $8 \mathrm{THz}$ ), suggesting that good phase-matching can be achieved by selecting a right optical pump wavelength. For LAPC, this wavelength occurs within the tuning range of our OPA, and for DAPC, the wavelength should be slightly longer. It should be stressed that a flat $\mathrm{THz}$ index is a general property for all amorphous EO polymers. Therefore, it can be envisioned that the use of an EO polymer with both a high EO coefficient and a good phase-matching property at the right optical wavelength is going to dramatically improve the performance of a $\mathrm{THz}$ system. Developing such an EO polymer is our goal.

We also developed a well-verified model for predicting the performance of polymer $\mathrm{THz}$ emitter-sensor pairs. Our modeling work not only confirmed that high EO coefficients and good phase-matching are essential for bright and broadband $\mathrm{THz}$ generation and detection, but also 
showed that low GVD in an EO polymer and well-shaped (close to transform limited) short optical pulses were also very important for that purpose. This model can be used as a guide to help select suitable $\mathrm{EO}$ polymers for $\mathrm{THz}$ applications.

However, using EO polymers as $\mathrm{THz}$ emitters and sensors is not without challenges. First, it is very demanding, even for a good organic chemist, to design an EO polymer that possesses all of the desired dielectric properties and yet can be easily processed. Even if it is possible to overcome these challenges, the thermal stability and heat damage threshold of EO polymers need to be improved significantly such that they can be used for a longer period. In fact, the latter issue also limits the wide employment of organic EO crystals as a slightly too strong pump can end the life of a very expensive crystal. Another newly opening question is how to control the $\mathrm{THz}$ absorption in an EO polymer. There does not exist any complete work in this area according to the literature. If guided-wave polymer $\mathrm{THz}$ emitters and sensors are to be developed, where a relatively long interaction-length is a "must," we have to first understand and solve this problem.

For freestanding polymer sensors, the lack of compatibility between the transverse poling configuration and the incident $\mathrm{THz}$ polarization partially negates the benefits that can result from high EO coefficients associated with the EO polymers. In-plane poling techniques can help address this issue.

For EO polymers to reach their full potential in the field of $\mathrm{THz}$ science and technology, it requires a close collaboration between the material scientist and optical scientists to tackle some of the challenges, if not all at one time.

\section{References and Notes}

1. E. R. Mueller, Phys. Today 27 (2003).

2. D. H. Auston, Appl. Phys. Lett. 3, 101 (1975).

3. D. H. Auston, K. P. Cheung, and P. R. Smith, Appl. Phys. Lett. 45, 284 (1984).

4. Y.-R. Shen, Prog. Quantum. Electron. 4, 207 (1976).

5. Q. Wu and X.-C. Zhang, Appl. Phys. Lett. 67, 3523 (1995).

6. A. Nahata, D. H. Auston, T. F. Heinz, and C. Wu, Appl. Phys. Lett. 68, 150 (1996).

7. X.-C. Zhang, X. F. Ma, Y. Jin, T.-M. Lu, E. P. Boden, P. D. Phelps, K. R. Stewart, and C. P. Yakymyshyn, Appl. Phys. Lett. 61, 3080 (1992).

8. T. J. Carrig, G. Rodriguez, T. S. Clement, A. J. Taylor, and K. R. Stewart, Appl. Phys. Lett. 66, 10 (1995).

9. T. J. Carrig, G. Rodriguez, T. S. Clement, A. J. Taylor, and K. R. Stewart, Appl. Phys. Lett. 66, 121 (1995).

10. J. J. Carey, R. T. Bailey, D. Pugh, J. N. Sherwood, F. R. Cruickshank, and K. Wynne, Appl. Phys. Lett. 81, 4335 (2002).

11. X. Zheng, A. Sinyukov, and L. M. Hayden, Appl. Phys. Lett. 87, 081115 (2005).

12. C. Soci and D. Moses, Synthetic Metals 139, 815 (2003).

13. O. Ostroverkhova, S. Shcherbyna, D. G. Cooke, R. F. Egerton, F. A. Hegmann, R. R. Tykwinski, S. R. Parkin, and J. E. Anthony, J. Appl. Phys. 98, 033701 (2005).

14. C. Fattinger and D. Grischkowsky, Appl. Phys. Lett. 53, 1480 (1988).
15. X.-C. Zhang, Y. Jin, and X. F. Ma, Appl. Phys. Lett. 61, 2764 (1992).

16. M. C. Nuss and J. Orenstein, Millimeter and Sub-millimeter Wave Spectroscopy in Solids, edited by G. Grüner, Springer, Berlin, (1998), pp. 7-50.

17. S. Kono, M. Tani, P. Gu, and K. Sakai, Appl. Phys. Lett. 77, 4104 (2000).

18. B. B. Hu, E. A. de Souza, W. H. Knox, J. Cunningham, and M. C. Nuss, Phys. Rev. Lett. 74, 1689 (1995).

19. S. Kono, M. Tani, and K. Sakai, Appl. Phys. Lett. 79, 898 (2001).

20. Y. C. Shen, P. C. Upadhya, E. H. Linfield, H. E. Beere, and A. G. Davies, Appl. Phys. Lett. 83, 3117 (2003).

21. D. Krokel, D. Grischkowsky, and M. B. Ketchen, Appl. Phys. Lett. 54, 1046 (1989).

22. U. D. Keil and D. Dykaar, Appl. Phys. Lett. 61, 1504 (1992).

23. C.-C. Wang, M. Curie, R. Sobolewski, and T. Y. Hsiang, Appl. Phys. Lett. 67, 79 (1995)

24. X. Zhou, T. Tang, L. S. Seah, C. J. Yap, and S. C. Choo, IEEE J. Quant. Electron. 34, 171 (1998).

25. Y. C. Shen, P. C. Upadhya, H. E. Beere, E. H. Linfield, A. G. Davies, I. S. Gregory, C. Baker, W. R. Tribe, and M. J. Evans, Appl. Phys. Lett. 85, 164 (2004).

26. D. H. Auston and M. C. Nuss, IEEE J. Quantum. Electron. 24, 184 (1988).

27. A. Nahata, D. Auston, C. Wu, and J. T. Yardley, Appl. Phys. Lett. 67, 1358 (1995).

28. K. Liu, H. S. Kang, T. K. Kim, and X. C. Zhang, Appl. Phys. Lett. 81, 4115 (2002).

29. C. Kubler, R. Huber, S. Tubel, and A. Leitenstorfer, Appl. Phys. Lett. 85, 3360 (2004).

30. J. Faure, J. Van Tilborg, R. A. Kaindl, and W. P. Leemans, Opt. Quant. Electron. 36, 681 (2004).

31. A. Nahata, A. Weling, and T. F. Heinz, Appl. Phys. Lett. 69, 2321 (1996).

32. G. Gallot, J. Zhang, R. W. McGowan, T.-I. Jeon, and D. Grischkowsky, Appl. Phys. Lett. 74, 3450 (1999).

33. G. Gallot and D. Grischkowsky, J. Opt. Soc. Am. B 16, 1204 (1999).

34. D. H. Auston and P. R. Smith, Appl. Phys. Lett. 41, 599 (1982).

35. J. A. Valdmanis, G. A. Mourou, and C. W. Gabel, Appl. Phys. Lett. 67, 211 (1982).

36. J. M. Wiesenfeld, IBM J. Res. Develop. 34, 141 (1990).

37. X. Zheng, W. S., R. Sobolewski, R. Adam, M. Mikulics, P. Kordos, and M. Siegel, Appl. Phys. Lett. 82, 2383 (2003).

38. J. Han, W. Zhang, W. Chen, L. Thamizhmani, A. K. Azad, and Z. Zhu, J. Phys. Chem. B 110, 1989 (2006).

39. W. J. Padilla, D. R. Smith, and D. N. Basov, J. Opt. Soc. Am. B 23, 404 (2005).

40. D. Grischkowsky, S. R. Keiding, M. van Exter, and C. Fattinger, J. Opt. Soc. Am. B 7, 2006 (1990).

41. D. M. Mittleman, M. Gupta, R. Neelamani, R. G. Baraniuk, J. V. Rudd, and M. Koch, Appl. Phys. B 68, 1085 (1999).

42. T. Ito, H. Shirakawa, and S. Ikeda, J. Polym. Sci. Chem. Ed. 12, 11 (1974).

43. P. B. Miranda, D. Moses, and A. Heeger, Phys. Rev. B 64, 081201 (2001).

44. D. Moses, A. Dogariu, and A. Heeger, Phys. Rev. B 61, 9373 (2000).

45. F. A. Hegmann, R. R. Tykwinski, K. P. H. Lui, J. E. Bullock, and J. E. Anthony, Phys. Rev. Lett. 89, 227403 (2002).

46. O. Ostroverkhova, D. G. Cooke, S. Shcherbyna, R. Egerton, F. A. Hegmann, R. R. Tykwinski, and J. E. Anthony, Phys. Rev. B 71, 035204 (2005).

47. O. Ostroverkhova, D. G. Cooke, F. A. Hegmann, J. E. Anthony, V. Podzorov, M. E. Gershenson, O. D. Jurchescu, and T. T. M. Palstra, Appl. Phys. Lett. 88, 162101 (2006).

48. R. Kersting, U. Lemmer, M. Deussen, H. J. Bakker, R. F. Mhart, H. Kurz, V. I. Arkhipov, H. Bassler, and E. O. Gobel, Phys. Rev. Lett. 73, 1440 (1994). 
49. W. Graupner, G. Cerullo, G. Lanzani, M. Nisoli, E. J. W. List, G. Leising, and S. D. Silvestri, Phys. Rev. Lett. 81, 3259 (1998).

50. E. Hendry, M. Koeberg, J. M. Schins, L. D. A. Siebbeles, and M. Bonn, Phys. Rev. B 70, 033202 (2004).

51. L. M. Hayden, A. M. Sinyukov, M. R. Leahy, J. French, P. Lindahl, W. Herman, R. J. Twieg, and M. He, J. Polym. Sci.:Part B: Polym. Phys. 41, 2492 (2003).

52. A. M. Sinyukov and L. M. Hayden, Opt. Lett. 27, 55 (2002).

53. A. M. Sinyukov and L. M. Hayden, J. Phys. Chem. B 108, 8515 (2004).

54. P. Y. Han, M. Tani, F. Pan, and X.-C. Zhang, Opt. Lett. 25, 675 (2000).

55. A. Schneider, I. Biaggio, and P. Gunter, Appl. Phys. Lett. 84, 2229 (2004).

56. F. Pan, G. Knöpfle, C. Bosshard, S. Follonier, R. Spreiter, M. S. Wong, and P. Günter, Appl. Phys. Lett. 69, 13 (1996).

57. R. Spreiter, C. Bosshard, F. Pan, and P. Gunter, Opt. Lett. 22, 564 (1997).

58. U. Meier, M. Bosch, C. Bosshard, F. Pan, and P. Gunter, J. Appl. Phys. 83, 3486 (1998).

59. M. Walther, J. K., S. R. Keiding, H. Takahashi, and H. Ito, Opt. Lett. 25, 911 (2000).

60. C. Bosshard, R. Spreiter, L. Degiorgi, and P. Gunter, Phys. Rev. B 66, 205107 (2002).

61. A. Schneider, I. Biaggio, and P. Gunter, Opt. Comm. 224, 337 (2003).

62. A. Schneider and P. Gunter, Appl. Opt. 45, 6598 (2006).

63. A. Schneider, M. Neis, M. Stillhart, B. Ruiz, R. U. A. Khan, and P. Gunter, J. Opt. Soc. Am. B 23, 1822 (2006).
64. K. Kawase, T. Hatanaka, H. Takahashi, K. Nakamura, T. Taniuchi, and H. Ito, Opt. Lett. 25, 1714 (2000).

65. K. Kawase, M. Mizuno, S. Sohma, H. Takahashi, T. Taniuchi, and H. Ito, Opt. Lett. 24, 1065 (1999).

66. Y. Takahashi, H. Adachi, T. Taniuchi, M. Takagi, Y. Hosokawa, S. Onzuka, S. Brahadeeswaran, M. Yoshimura, Y. Mori, and H. Masuhara, J. Photochem. Photobiol. A 183, 247 (2006).

67. M. C. Beard, G. M. Turner, and C. A. Schmuttenmaer, J. Am. Chem. Soc. 122, 11541 (2000).

68. M. Nagai, K. Tanaka, H. Ohtake, T. Bessho, T. Sugiura, T. Hirosumi, and M. Yoshida, Appl. Phys. Lett. 85, 3974 (2004).

69. T.-D. Kim, J. Luo, Y. Tian, J.-W. Ka, N. M. Tucker, M. Haller, J. W. Kang, and A. K.-Y. Jen, Macromolecules 39, 1676 (2006).

70. J. W. Kang, T. D. Kim, J. Luo, M. Haller, and A. K. Y. Jen, Appl. Phys. Lett. 87, 071109 (2005).

71. T.-D. Kim, J. Luo, J.-W. Ka, S. Hau, Y. Tian, Z. Shi, N. M. Tucker, S. H. Jang, J.-W. Kang, and A. K.-Y. Jen, Adv. Mater. 18, 3038 (2006).

72. H. Cao, R. A. Linke, and A. Nahata, Opt. Lett. 29, 1751 (2004).

73. N. C. J. van der Valk, P. C. M. Planken, A. N. Buijserd, and H. J. Bakker, J. Opt. Soc. Am. B 22, 1714 (2005).

74. T. Taniuchi, S. Okada, and H. Nakanishi, J. Appl. Phys. 95, 5984 (2004).

75. www.rainbowphotonics.com.

76. L. Xu, X.-C. Zhang, and D. H. Auston, Appl. Phys. Lett. 61, 1784 (1992).

77. X. Zheng, C. V. McLaughlin, M. R. Leahy-Hoppa, A. M. Sinyukov, and L. M. Hayden, J. Opt. Soc. Am. B 23, 1338 (2006).

Received: XX Xxxxxx XXXX. Revised/Accepted: 25 January 2007. 